\title{
Disome-seq reveals widespread ribosome collisions that promote cotranslational protein folding
}

Taolan Zhao ${ }^{1,2^{*}+}$, Yan-Ming Chen ${ }^{1,2,3 \dagger}$, Yu Li $i^{4,5 \dagger}$, Jia Wang ${ }^{1,2,3}$, Siyu Chen ${ }^{1,2,3}$, Ning Gao ${ }^{5^{*}}$ and Wenfeng Qian ${ }^{1,2,3^{*}}$ (D)

\author{
*Correspondence: tlzhao@genetics. \\ ac.cn; gaon@pku.edu.cn; wfaian@ \\ genetics.ac.cn \\ 'Taolan Zhao, Yan-Ming Chen and \\ Yu Li contributed equally to this \\ work. \\ 'State Key Laboratory of Plant \\ Genomics, Institute of Genetics and \\ Developmental Biology, Innovation \\ Academy for Seed Design, Chinese \\ Academy of Sciences, Beijing \\ 100101, China \\ ${ }^{5}$ State Key Laboratory of Membrane \\ Biology, Peking-Tsinghua Center for \\ Life Sciences, School of Life \\ Sciences, Peking University, Beijing \\ 100871, China \\ Full list of author information is \\ available at the end of the article
}

\begin{abstract}
Background: The folding of proteins is challenging in the highly crowded and sticky environment of a cell. Regulation of translation elongation may play a crucial role in ensuring the correct folding of proteins. Much of our knowledge regarding translation elongation comes from the sequencing of mRNA fragments protected by single ribosomes by ribo-seq. However, larger protected mRNA fragments have been observed, suggesting the existence of an alternative and previously hidden layer of regulation.
\end{abstract}

Results: In this study, we performed disome-seq to sequence mRNA fragments protected by two stacked ribosomes, a product of translational pauses during which the 5'-elongating ribosome collides with the 3'-paused one. We detected widespread ribosome collisions that are related to slow ribosome release when stop codons are at the A-site, slow peptide bond formation from proline, glycine, asparagine, and cysteine when they are at the P-site, and slow leaving of polylysine from the exit tunnel of ribosomes. The structure of disomes obtained by cryo-electron microscopy suggests a different conformation from the substrate of the ribosome-associated protein quality control pathway. Collisions occurred more frequently in the gap regions between ahelices, where a translational pause can prevent the folding interference from the downstream peptides. Paused or collided ribosomes are associated with specific chaperones, which can aid in the cotranslational folding of the nascent peptides.

Conclusions: Therefore, cells use regulated ribosome collisions to ensure protein homeostasis.

Keywords: Translation elongation, Disome-seq, Ribosome collision, Translational pause, Ribosome release, Ribosome-associated chaperones, Cotranslational protein folding, Protein homeostasis, Disome structure

\section{Background}

Translation elongation is a crucial process through which the genetic information in a transcript is sequentially decoded into a peptide chain by ribosomes. Yet, the mRNA sequence of coding regions can harbor more information than the amino-acid sequence [1]; the local rate of translation elongation is non-uniform and fine-tuned [2,

(c) The Author(s). 2021 Open Access This article is licensed under a Creative Commons Attribution 4.0 International License, which permits use, sharing, adaptation, distribution and reproduction in any medium or format, as long as you give appropriate credit to the original author(s) and the source, provide a link to the Creative Commons licence, and indicate if changes were made. The images or other third party material in this article are included in the article's Creative Commons licence, unless indicated otherwise in a credit line to the material. If material is not included in the article's Creative Commons licence and your intended use is not permitted by statutory regulation or exceeds the permitted use, you will need to obtain permission directly from the copyright holder. To view a copy of this licence, visit http://creativecommons.org/licenses/by/4.0/. The Creative Commons Public Domain Dedication waiver (http://creativecommons.org/publicdomain/zero/1.0/) applies to the data made available in this article, unless otherwise stated in a credit line to the data. 
3]. Programmed variation in elongation rate may participate in the regulation of protein folding $[2,4,5]$, which is challenging within the crowded and sticky cellular environments $[6,7]$. A change in the rate of translation elongation can result in protein misfolding $[2,5]$, which further leads to developmental abnormalities, neurologic diseases, and cancers [8].

Despite the importance of translation elongation, it has been mainly studied with heterologous reporter genes [9-11]. A strong ribosomal stalling signal was often placed in these reporter genes; the $5^{\prime}$-elongating ribosome collides with the stalled ribosome, leading to a di-ribosome (we hereafter refer such stacked ribosomes induced by a strong ribosomal stalling signal in heterologous reporters as to di-ribosomes) or even tri-ribosome. Structure analyses indicated that di-ribosomes and tri-ribosomes were often unable to resume translation and can trigger the ribosome-associated protein quality control (RQC) pathway [9, 12-16].

The knowledge of the causes for endogenous translational pausing remains highly limited, mainly because the detection of translational pauses is technically challenging in endogenous genes. The development of ribo-seq, an approach that sequences ribosome-protected mRNA fragments at codon resolution, significantly increased our knowledge on translation elongation [17, 18]. Accumulation of ribosome footprints at a site indicates slow translation elongation (i.e., a translational pause); based on this idea, sequence determinants of translation elongation have been discovered, such as synonymous codon usage [19-21], positively charged nascent peptides [22], and mRNA secondary structures [23]. However, traditional riboseq misses the information of ribosome collisions [24, 25]. Instead, ribosome collisions can be studied by sequencing the mRNA fragments protected by disomes, which refer to endogenous stacked ribosomes in this study. Disomes were detectable by sucrose gradient centrifugation [26] and were observed in faulty mRNAs or 3 '-untranslated regions [24]. However, the genomic landscape and the sequence determinants of endogenous ribosome collisions remain largely unknown in the coding sequences of faithfully transcribed mRNAs.

The consequence of endogenous translational pausing also remains unclear. It has been suggested that translation elongation can regulate cotranslational protein folding $[2,4,5$, 27, 28]. For example, accumulating evidence supported that the CAG expansion in Huntington's disease led to incorrect translational pausing and thereby improper folding of the signal peptide for the subcellular localization of the Htt protein [29]. Non-optimal codons formed clusters during evolution [30,31]; they may create slow-translation regions and participate in protein folding [32]. However, the mechanisms by which translational pauses regulate cotranslational protein folding remain understudied.

In this study, we captured the mRNA footprints protected by two stacked ribosomes in fast proliferating yeast cells. Such data provide a chance to reveal the translational dynamics that are undetectable by the traditional ribo-seq (i.e., monosome-seq). We identify the sequence features that are associated with ribosome collisions and validate some features with reporter genes. Cryo-electron microscopy (cryo-EM) analyses indicate that the majority of endogenous ribosome collisions form a different structure from the RQC-inducing di-ribosomes. With bioinformatics analyses, we show that ribosome pauses or collisions tend to take place in the gap regions between $\alpha$-helices. In fact, paused or collided ribosomes 
are often associated with specific chaperones that can assist protein folding, as indicated by mass spectrometry analyses. As a consequence, a nascent peptide is ready to be correctly folded during translation.

\section{Results}

\section{Translational pauses generate disomes from collisions of ribosomes}

Most mRNAs are associated with multiple ribosomes [33], and the speed of translational elongation varies, with some events such as tRNA depletion, known to cause ribosomes to slow down and even to pause translation [8]. We therefore hypothesized that a slowdown or pause of one leading ribosome might generate a collision between the paused ribosome and the 5 '-elongating ribosome. These collisions would result in a single RNase I resistant fragment with approximately twice the footprint length of a single ribosome (Fig. 1a).

To test this idea, we extracted ribosome-bound mRNA from exponentially dividing yeast cells, digested the unprotected mRNA using RNase I, and performed sucrose gradient ultracentrifugation to separate particles of different densities. In addition to the abundant monosome particles, we observed a significant amount of particles whose density was the same as that of pre-digestion transcripts bound by two ribosomes, suggesting that the particle contains two ribosomes (Fig. 1b). These disomes persisted with increased RNase I concentration, indicating that they are not the result of incomplete digestion (Additional file 1: Fig. S1). We estimated the relative abundance of monosomes and disomes from the area ratio of the monosome and disome fractions in the profile. The ratio was approximately 16.3:1 (Fig. 1b, right panel), suggesting that the population ratio of monosomes and disomes is $32.6: 1$. In other words, $(1 /(16.3+1)=) 5.8 \%$ ribosomes are trapped in disomes in fast-proliferating yeast cells.

To determine if disomes were caused by paused ribosomes, we induced translational pauses at histidine codons by growing yeast in a low dose of 3-amino-1,2,4-triazole (3AT), an inhibitor of histidine biosynthesis [34]. We performed high-throughput sequencing on monosome (monosome-seq) and disome (disome-seq) fragments (Fig. 1c, Additional file 1: Fig. S2a and Table S1-3) developed in previous studies [17, 24]. Consistent with previous observations [17], in-frame 28-nucleotide (nt) footprints were most abundant in the monosome library (Additional file 1: Fig. S3a). In contrast, the most abundant footprints in disome-seq were 58 and 59-nt when cells were treated with 3-AT, with the 58-nt footprints being in-frame (Fig. 1d). As a negative control, randomly fragmented $\sim 28$-nt mRNA-seq reads did not display any 3-nt periodicity (Additional file 1: Fig. S3b).

During histidine starvation, ribosomes should be paused when the histidine codon CAC or CAT is at the A-site, where decoding takes place. Consistently, the main peak in the 28-nt monosome footprints (the $5^{\prime}$-end) was 15-nt upstream of the histidine codons (Fig. 1e). The main peak of the 58-nt disome footprints was 45-nt upstream of the histidine codons, 30-nt upstream of the 15-nt peak from the monosome footprints (Fig. 1e). This 30-nt spacing between the two peaks perfectly fits one in-frame ribosome, suggesting that the 58-nt disome footprints were composed of two collided ribosomes of which the 3'-leading one was paused. The 


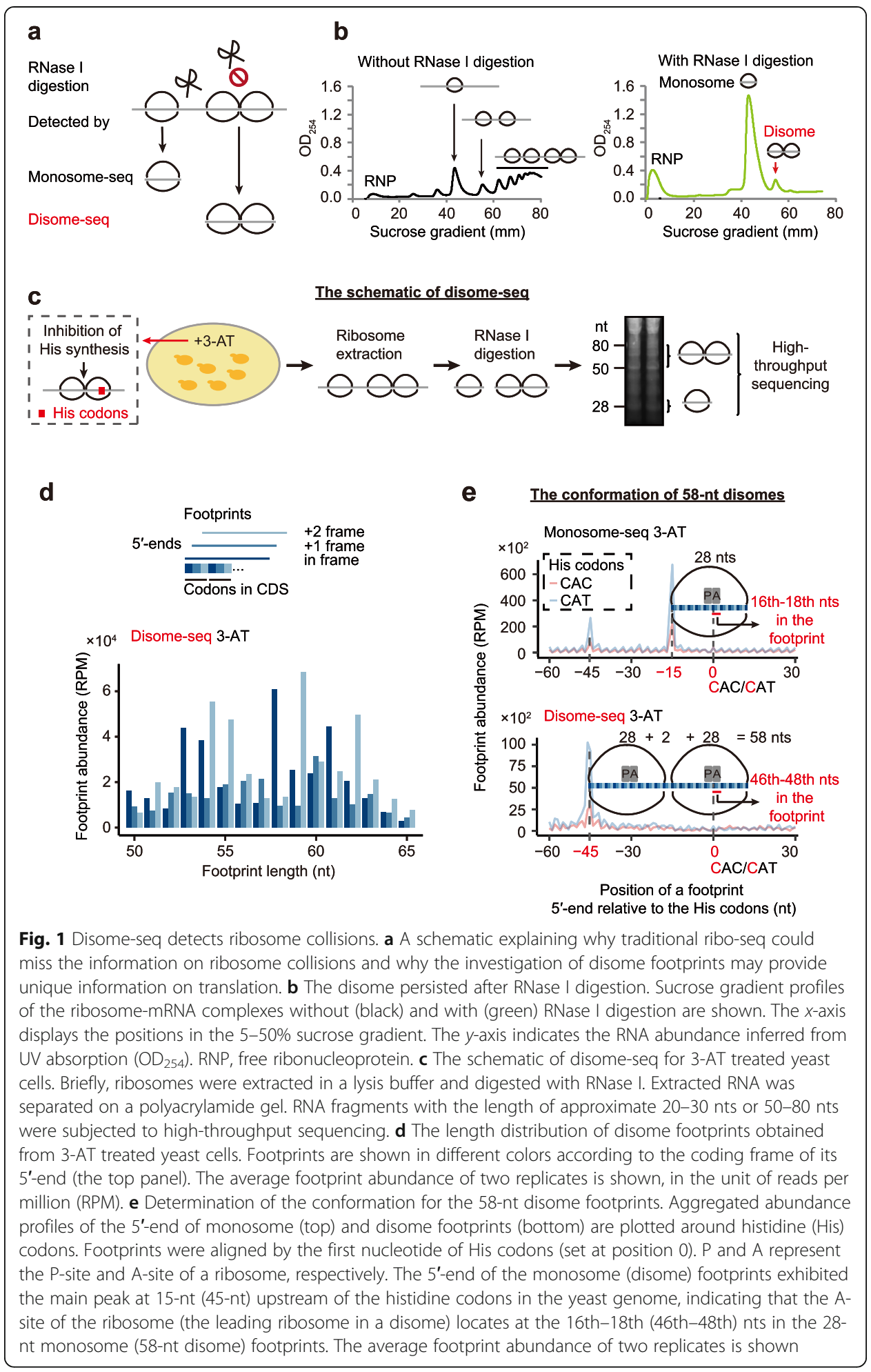




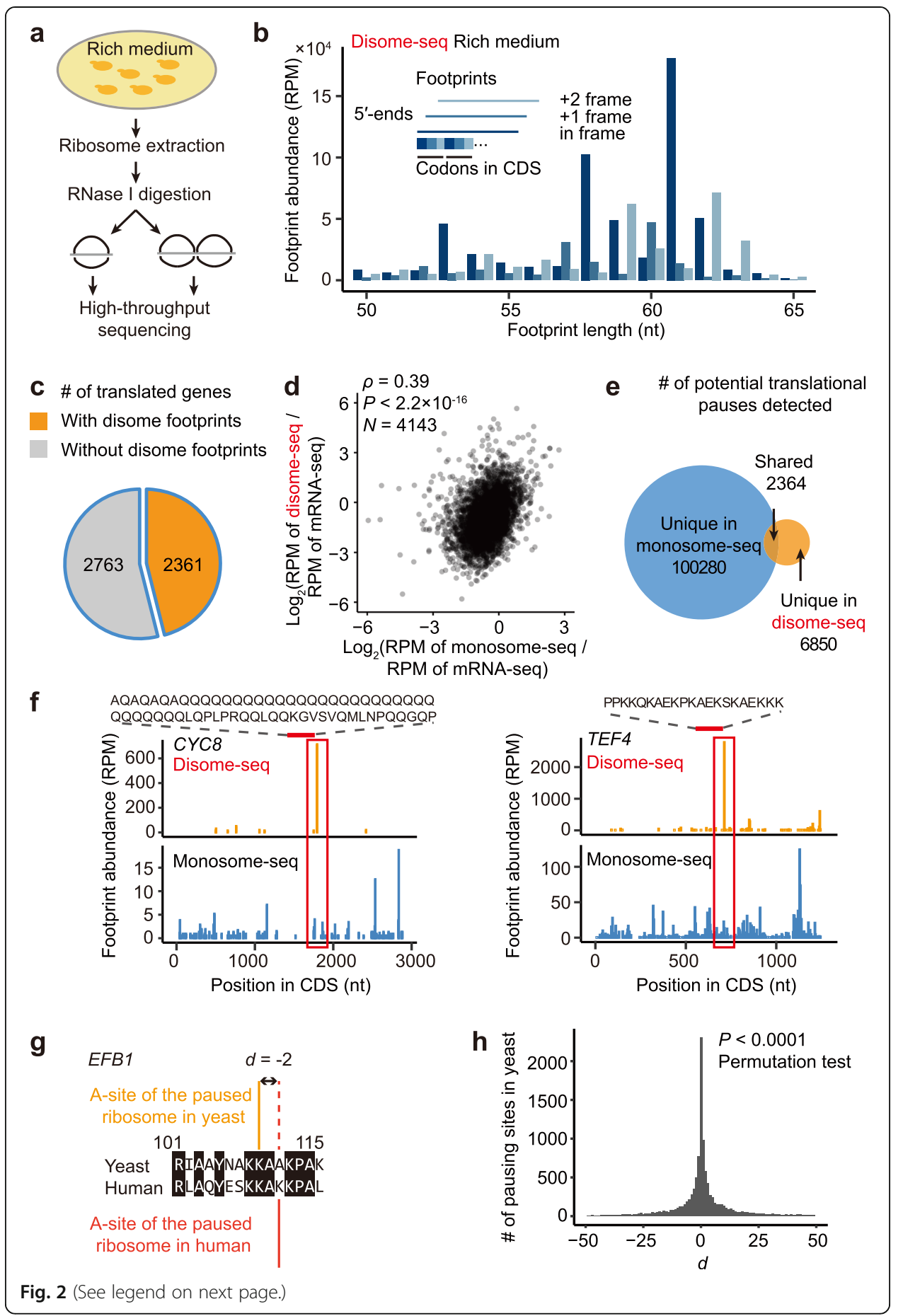


(See figure on previous page.)

Fig. 2 Disome-seq detects the translational pauses missed in monosome-seq. a The schematic of disome-seq for yeast cells cultivated in the rich medium. $\mathbf{b}$ The length distribution of disome footprints obtained from yeast cells cultivated in the rich medium. Similar to Fig. 1d. c Widespread ribosome collisions were detected in the yeast genome. Numbers of translated genes (genes with at least one monosome footprint) with and without disome footprints are shown in orange and gray, respectively. Footprints in two biological replicates were combined. 589,461 monosome footprints were used to define translated genes, and the disome-seq footprints were down-sampled to 18,082 footprints in order to match the population ratio between monosomes and disomes (32.6:1). d More frequent ribosome collision was observed in the gene with higher monosome density. e The translational pauses detected from monosome footprints (blue) and disome footprints (orange) rarely overlapped. Only the codon site with the footprint abundance greater than the mean of the corresponding gene was considered as a translational pause. If the A-site of a monosome footprint overlaps the A-site of the leading ribosome of a disome footprint, the translational pause is considered as "shared." The disome-seq reads were down-sampled as in Fig. 2c. f Two genes exemplify the unique information of translational pauses obtained by disome-seq. The A-site of a monosome footprint (blue) or that of the leading ribosome of a disome footprint (orange) is shown along the coding sequence (CDS) of CYC8 and TEF4. $\mathbf{g}$ The schematic of estimating the distance ( $d$ ) to the closest human pause for a yeast pause. $\mathbf{h}$ The distribution of $d$ between yeast and humans. The $P$ value was given by the permutation test

sharp peak in Fig. 1e also indicates that disome-seq detects ribosome collisions at codon resolution.

\section{Disome-seq enables detection of widespread translational pauses which cannot be identified via monosome sequencing}

To determine the genomic locations of ribosome collisions in fast-proliferating cells, we performed disome-seq, monosome-seq, and mRNA-seq for yeast cells growing in the mid-log phase in the rich medium (Fig. 2a, b, Additional file 1: Fig. S2b, S3c-f, and Table S1-S3). Ribosome collisions were observed in 2361 out of the 5124 translated genes (46\%, Fig. 2c). This proportion remained substantial $(24 \%, 1156 / 4742)$ when we applied a more stringent criterion-a gene with collision was called when it was supported by at least three unique molecular identifiers (UMIs, used to exclude PCR duplicates) in each biological replicate of disome-seq. In general, genes harboring more ribosomes per mRNA per unit length (i.e., ribosome density, inferred from monosomeseq) tended to exhibit higher frequency of ribosome collisions (per mRNA per unit length, $\rho=0.39, P<2.2 \times 10^{-16}, N=4143$, Spearman's correlation, Fig. $2 \mathrm{~d}$ ). These observations indicate widespread ribosome collisions in unstressed cells.

There are two possibilities regarding ribosome collisions inside open reading frames and the relation between translational pauses identified in monosome-seq vs. disomeseq. The first is that sites with high monosome footprint abundance identify all paused ribosomes in the cell and that the collisions identified in disome-seq are simply a subset of them. In this case, it is likely that the upstream ribosome is far $5^{\prime}$ of the paused ribosome, and by the time the upstream ribosome approaches, most paused ribosome has resumed elongation. In this model, all translational pauses identified by disome-seq will also be identified by monosome-seq. The second possibility is that disome-seq captures translational pauses that occur at locations not identified by monosome sequencing, possibly because the collision often occurs not long after the pause of the leading ribosome.

To differentiate these two possibilities, we measured the intersection of the translational pausing events from the two methods. Disome-seq often identified translational 
pauses that are undetectable in monosome-seq (Fig. 2e, f). For example, CYC8 is a gene encoding a general transcriptional co-repressor that can fold as the prion [OCT+] [35]; a translational pause downstream of polyglutamine (polyQ) was detected by disomeseq instead of monosome-seq (Fig. 2f). Similarly, TEF4p, the $\gamma$ subunit of the elongation factor eEF1B, contains a lysine-rich region that is often ubiquitinylated or succinylated [36, 37]; the downstream translational pause was uniquely detected by disome-seq (Fig. 2f). The inability of monosome-seq to fully characterize the dynamics of the ribosome along the mRNA is likely due to the omission of the disome protected mRNA fragments (Fig. 1a). Consistently, while the monosome or disome density correlated well among genes between biological replicates, the correlation between the monosome and disome densities was much weaker (Additional file 1: Fig. S2).

To determine if ribosome collisions are likely functional and thereby are conserved over evolution, we retrieved the disome-seq data generated for human cells in a recent study [38]. We projected disome footprints detected in human cells to the orthologous positions of the yeast genome based on the amino acid at the A-site of the paused ribosome. For each ribosome collision in yeast, we estimated its distance $(d)$ to the closest counterpart in human cells (Fig. 2g). $d$ was equal to zero in $19.4 \%$ of yeast ribosome collision sites (Fig. $2 \mathrm{~h}$ ), indicating that these yeast ribosome collisions occur at the same site in the human genome. To assess the statistical significance, we assigned ribosome collisions to random positions in human cells 10,000 times, keeping the number of ribosome collisions in each gene unchanged. The fraction of conserved ribosome collisions $(d=0)$ in every randomized sample was smaller than what we observed in the genomes $(P<0.0001$, the permutation test, Fig. $2 \mathrm{~h})$, indicating that the positions of ribosome collisions are evolutionarily conserved over the 1300-million-year evolution between yeast and humans [39].

\section{Ribosomes tend to collide at stop codons}

Visually, we noticed that many highly expressed genes exhibited large numbers of disome-seq reads at the stop codon, but also at internal codons, suggesting that ribosome collisions occur both internally and at the ends of open reading frames (Fig. 3a). To identify the causes of these collisions, we searched for the sequence features at the A-site, P-site, and exit tunnel of the 3'-paused ribosome of a disome, respectively. We defined the propensity of a codon to induce ribosome collisions at the A-site (i.e., the A-site pausing score) for each of the 64 codons as its enrichment in the disome footprints (Fig. 3b). Taking the codon GAA as an example (Fig. 3b), all disome fragments from one gene were classified into two categories based on the codon identity at the Asite, GAA or the others. The odds ratio was estimated for each gene with the corresponding codon frequency in the appendant mRNA-seq as the background; the A-site pausing score was defined as the common odds ratio across all genes calculated by Mantel-Haenszel tests (Fig. 3b). A significantly $>1$ A-site pausing score implies slow decoding of the codon.

To test if the A-site pausing score defined here can effectively detect the slow decoding of the histidine codons in cells treated with 3-AT, we estimated the A-site pausing score for each codon in yeast cells treated with 3-AT or growing in the rich medium. 


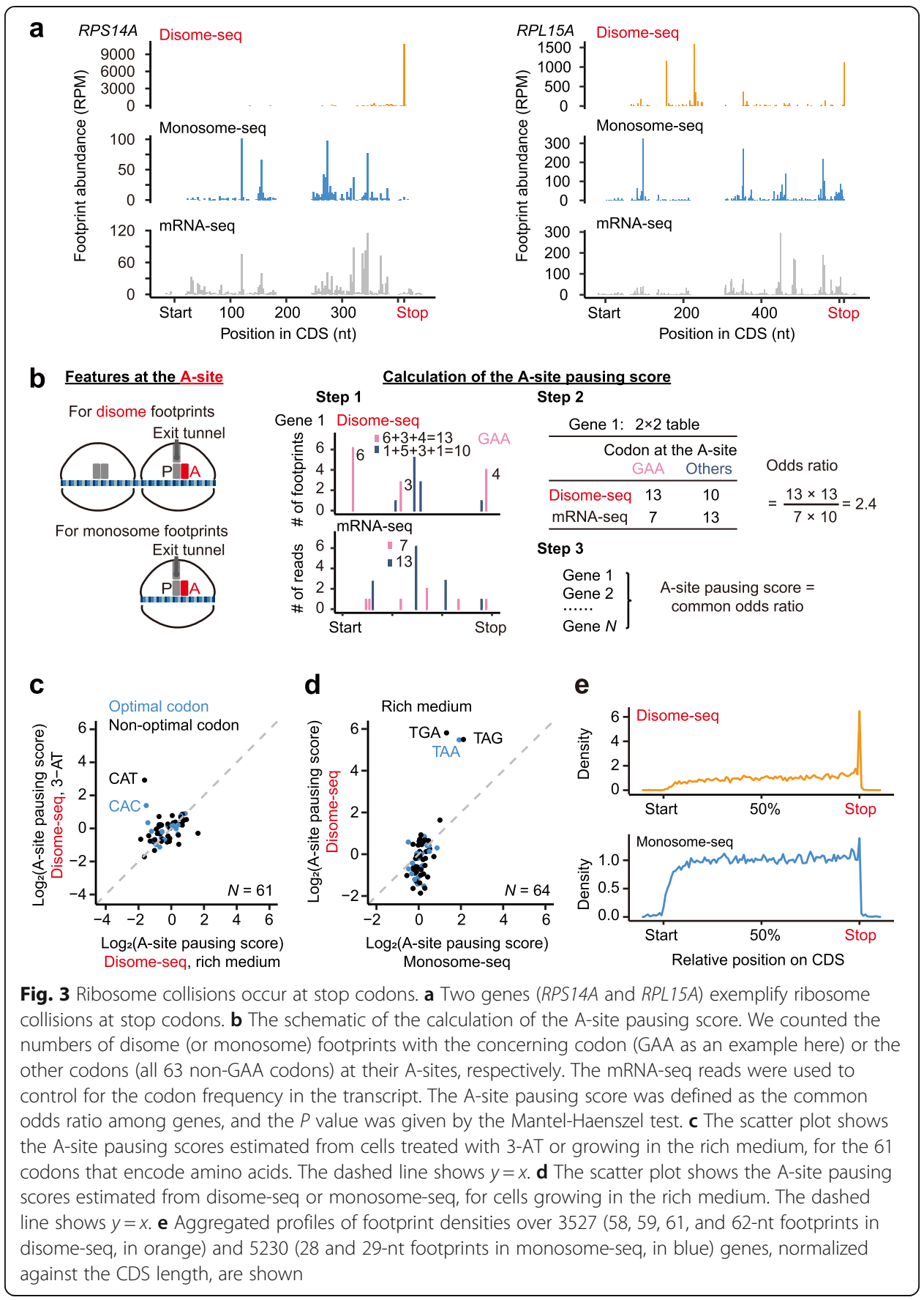

Two histidine codons deviated from the diagonal line (Fig. 3c), indicating that the pausing score defined in this study is useful to detect translational pauses.

In comparing the disome-seq and the monosome-seq data for cells growing in the rich medium, all three stop codons exhibited extremely high A-site pausing scores in disome-seq, while their A-site pausing scores in monosome-seq were only a bit higher than the amino-acid coding codons' (Fig. 3d). The results were consistent between two biological replicates of disome-seq (Additional file 1: Fig. S4a). To avoid artifacts generated by PCR amplification bias during the preparation of the high-throughput sequencing library, we used UMI to exclude PCR duplicates. Three stop codons still exhibited 
a

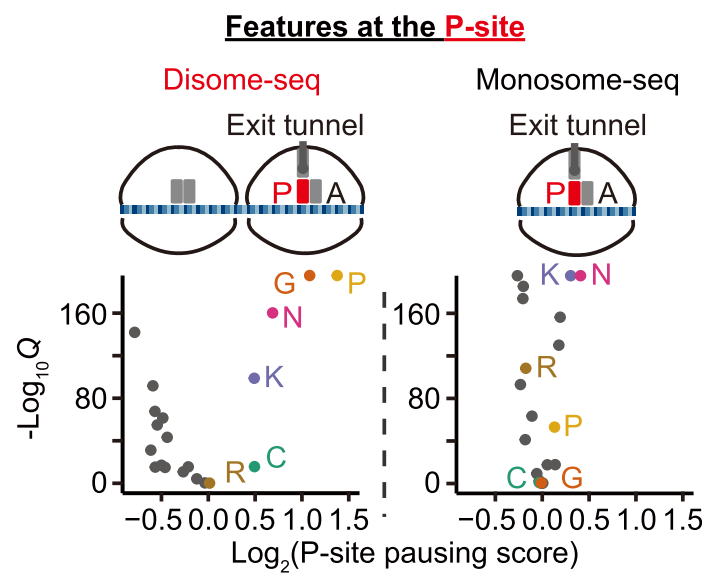

b

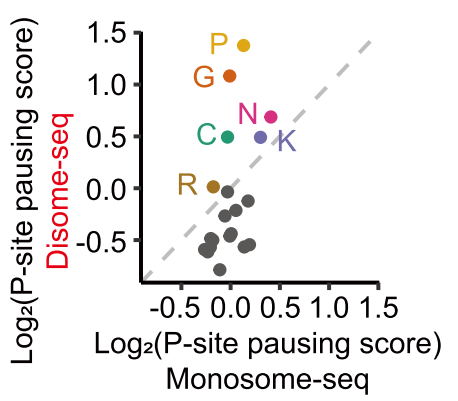

C

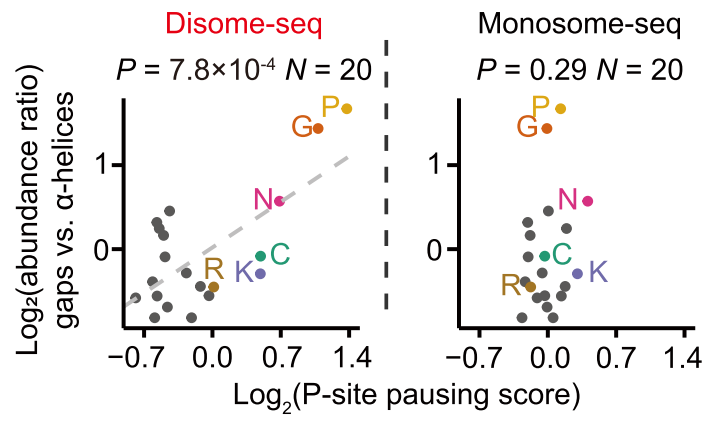

d

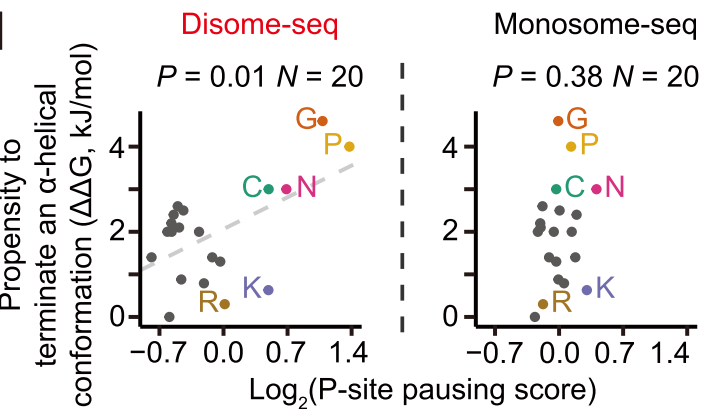

Fig. 4 (See legend on next page.) 
(See figure on previous page.)

Fig. 4 Ribosome collisions occur at the amino acids that can terminate a-helices. a Volcano plots show the P-site pausing score (based on disome and monosome footprints, respectively) of each amino acid and the corresponding $Q$ value (false discovery rate). Six amino acids with $>1$ P-site pausing scores in disome-seq are labeled. P, proline; $G$, glycine; N, asparagine; K, lysine; C, cysteine; R, arginine. $\mathbf{b}$ The scatter plot shows the P-site pausing scores estimated from disome-seq or monosome-seq. The dashed line shows $y=x$. c The scatter plots show the P-site pausing score of an amino acid and the ratio of the whole-genome occurrence of the amino acid in gaps vs. in a-helices. The $P$ value was given by linear regression. The dashed line represents the linear regression line (if statistically significant). The residuals of both linear models followed the normal distribution ( $P=0.63$ and $P=0.80$, the Kolmogorov-Smirnov tests). d The scatter plots show the P-site pausing score of an amino acid and the propensity to terminate an a-helix by the amino acid ( $\Delta \mathrm{G}$ relative to alanine, in the unit of $\mathrm{k} / \mathrm{mol}$ ). The $P$ value was given by linear regression. The dashed line represents the linear regression line (if statistically significant). The residuals of both linear models followed the normal distribution ( $P=0.25$ and $P=0.89$, the Kolmogorov-Smirnov tests)

extremely high A-site pausing scores (Additional file 1: Fig. S4b). Consistently, disome reads accumulated at stop codons at the genomic scale (Fig. 3e). Collectively, these observations suggest that ribosome release is slow.

\section{Ribosome collisions occur preferentially at the amino acids that terminate a-helices}

To determine if peptide-bond formation causes ribosome collisions, we similarly calculated the P-site pausing score for each of the 20 amino acids; amino-acid identity, but not codon identity, is likely to matter at the P-site. Five amino acids (proline, glycine, asparagine, cysteine, and lysine) showed significantly $>1$ P-site pausing scores in disome-seq (Fig. 4a, b). Among them, proline and glycine are known poor substrates for the formation of a peptide bond [40] and therefore slow down translation elongation and lead to ribosome collisions. It remains unclear how other amino acids induce ribosome collisions. Nevertheless, these amino acids share the same property that they weaken the stability of the $\alpha$-helix [41]. Proline and glycine are conformationally either too inflexible or too flexible, respectively, to form an $\alpha$-helix. The bulk and shape of asparagine and cysteine also destabilize $\alpha$-helices. Positively charged residues (e.g., polylysine) likely repel each other and prevent the formation of an $\alpha$-helix [41].

We estimated the number of occurrences of each amino acid in gaps or $\alpha$-helices in the yeast genome; the ratio between them indicates the propensity of an amino acid to appear in the gap. We found that this ratio could be predicted from the P-site pausing score of the amino acid in disome-seq $\left(P=7.8 \times 10^{-4}, N=20\right.$, linear regression, Fig. 4c) but not in monosome-seq $(P=0.29, N=20$, Fig. 4c). Furthermore, the propensity to terminate an $\alpha$-helical conformation of an amino acid (measured by $\Delta \Delta G$ ) [42, 43] was also predictable from the P-site pausing score in disome-seq $(P=0.01, N=20$, linear regression, Fig. $4 \mathrm{~d})$ but not in monosome-seq $(P=0.38$, Fig. $4 \mathrm{~d})$, suggesting a sequencemediated coupling between ribosome collisions and the intrinsically disordered region.

Ribosome collisions tend to occur when the nascent polylysine chain is in the exit tunnel Positively charged nascent peptides can hinder translation elongation by interacting with the negatively charged exit tunnel [22]. To test this hypothesis with disome-seq data, we calculated the exit-tunnel pausing score from the enrichment of each of the $\left(20^{3}=\right) 8000$ amino-acid 3-mers in the 20-amino-acid region upstream of the P-site of the leading ribosome. Taking triple-lysine as an example (Fig. 5a), a window of three amino acids slid one amino acid per step in this 21-amino-acid region, counting the 


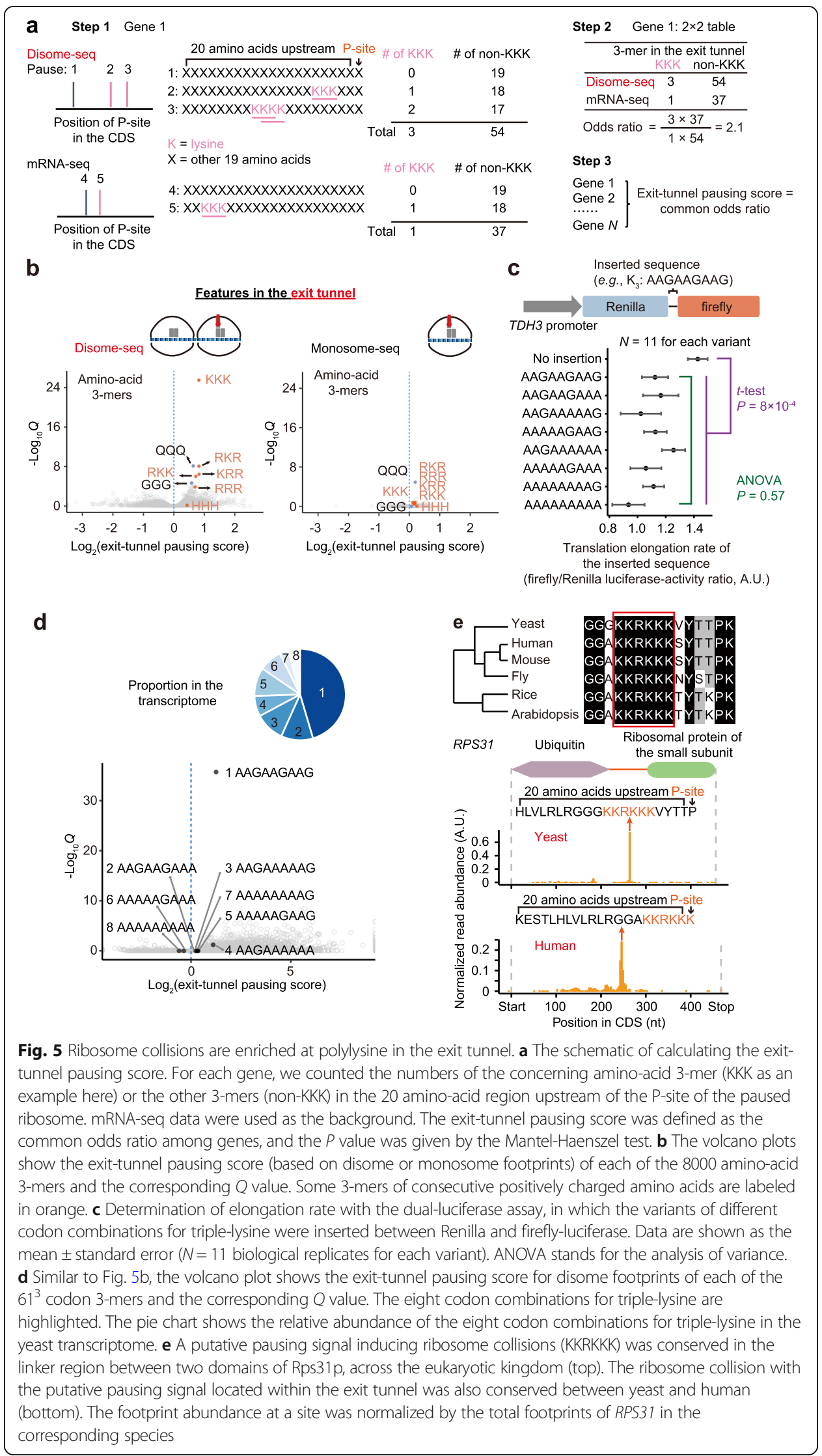


number of triple-lysine or the total number of all other 3-mers for each pausing site; these numbers were added together for all pausing sites of a gene. The odds ratio was estimated for each gene with the corresponding numbers in the appendant mRNA-seq data as the background. The exit-tunnel pausing score was defined as the common odds ratio across the genome aggregated by the Mantel-Haenszel test.

Ribosome collisions were strongly associated with positively charged 3-mers (e.g., RKR) when in exit tunnels; by contrast, such pausing signals were obscured in monosome-seq (Fig. 5b). Triple-lysine had the strongest collision signal among all the 3-mers (Fig. 5b), much stronger than other positively charged 3-mers (e.g., RKR, RKK, $\mathrm{KRR}$, and RRR). It has been suggested that the reading-through of stop codons could result in the translation of poly(A) tails into polylysine, which activates the RQC pathway [44]. As a result, A-tracts are often used to induce RQC in heterologous reporter systems [10]. Since that AAA is the non-optimal codon of lysine [45], it is unclear if its poor codon optimality also participates in promoting translational pausing in these experiments.

To experimentally determine if combinations of synonymous codons for triple-lysine have different efficacies to induce translational pauses, we constructed a dual-luciferase reporter system by inserting individual synonymous variants of triple-lysine between the Renilla and firefly-luciferases (Fig. 5c). A reduction in the firefly/Renilla luciferaseactivity ratio can report translational pausing induced by the inserted sequence-translation elongation replaces initiation as the rate-limiting step in protein synthesis of the downstream luciferase. We did not detect significant differences in the firefly/Renilla ratio among the eight synonymous codon combinations of triple-lysine $(P=0.57$, analysis of variance, Fig. 5c). In contrast, the firefly/Renilla ratio was significantly reduced when triple-lysine was inserted ( $P=8 \times 10^{-4}$, the two-tailed $t$ test, Fig. 5c), suggesting that lysine residues, instead of the non-optimal AAA codons, are accountable for the ribosome collisions in A-tracts.

This observation is not unexpected because it is the identity of amino acids rather than the identity of synonymous codons that should matter once the nascent peptide is in the exit tunnel. Consistently, when we separately estimated the pausing scores for the eight synonymous codon combinations encoding triple-lysine (Fig. 5d), endogenous ribosome collisions induced by triple-lysine were mainly contributed by triple-AAG (Fig. 5d), the most abundant codon combination for triple-lysine in the yeast transcriptome (Fig. 5d).

Polylysines often appear in the gap regions because the repellence among the positively charged residues prevents the formation of an $\alpha$-helix [41]. For example, polylysine-induced ribosome collision occurs in the linker between the two domains of RPS31p, the fusion protein of ubiquitin and ribosomal protein (Fig. 5e), raising the possibility that a translational pause benefits the cell by providing sufficient time for the cotranslational folding of the upstream domain. Consistently, despite being in an intrinsically disordered region, these positively charged amino acids (KKRKKK) are conserved across eukaryotes (Fig. 5e). To test if the ribosome collision is also conserved, we retrieved the disome-seq data for human cells reported in a recent study [38]. The ribosome collision, with these positively charged nascent peptides in the exit tunnel, was detected in both human and yeast cells (Fig. 5e), albeit these two species have diverged since 1300 million years ago [39], suggesting a function of this inter-domain ribosome collision. 


\section{Disomes exhibit a different structure from the RQC-inducing di-ribosomes}

The pervasiveness of ribosome collisions detected in fast-proliferating cells (Fig. 2) and the ubiquitous sequence signals of disomes (e.g., proline and polylysine, Figs. 4 and 5) in endogenous genes are astonishing since the stacked ribosomes observed previously in reporter genes (di-ribosomes) could lead to the decay of both mRNA and nascent peptides through the no-go decay and the RQC pathways [46]. Alternatively, some disome footprints detected in fast proliferating cells may simply reflect the inevitable nature of stochastic and temporary ribosome collisions since an mRNA is often translated simultaneously by multiple ribosomes. For example, in the top $2 \%$ of highly translated mRNAs, on average $\sim 3$ ribosomes were located in a 100-nt region (Additional file 1: Fig. S5), a highly crowded situation for ribosomes.

Consistent with this idea, collision propensity of a transcript was positively correlated with ribosome density (Fig. 2d) - the 3'-paused ribosomes are easier to be caught up by a 5 '-elongating ribosome on mRNAs with high ribosomal flux. We speculate that these ribosome collisions were neither induced by faulty mRNA nor by aberrant nascent peptides, and it would be very costly for cells to activate the no-go decay or the RQC pathway for these ribosome collisions. Their translation is likely transiently interrupted and to be resumed. If true, we predict that these disomes should be structurally different from the RQC-inducing di-ribosomes reported in previous studies.

To test if the disomes collected from endogenous genes in fast-proliferating cells are structurally identical to the RQC-inducing di-ribosomes, we performed cryo-EM analyses of disomes collected after RNase I digestion. Following the previous protocols for the structural determination of di-ribosomes or tri-ribosomes $[9,15,16]$, we omitted the elongation inhibitor cycloheximide in the lysis buffer. Disomes showed a structure of two ribosomes linked by a bent mRNA, with two $40 \mathrm{~S}$ subunits facing toward each other (Fig. 6a). Furthermore, the $5^{\prime}$-trailing ribosomes were detected in the rotated state that harbors hybrid A/P and P/E tRNAs (Fig. 6a), indicating that they cannot further translocate, likely due to the road-blocking 3 '-leading ribosomes. These observations are in general consistent with the structure of di-ribosomes induced by hard stalls in cell-free systems $[9,15,16]$.

However, two distinct features indicate the essential difference between disomes and di-ribosomes. First, the relative orientation between the two ribosomes in the disome was different from that in the RQC-triggering di-ribosome (Fig. 6b). It was reported that the two ribosomes in the di-ribosome form a large interface with highly specific contacts between two $40 \mathrm{~S}$ subunits (Additional file 1: Fig. S6a), which was considered to be a feature recognizable by the RQC initiation factors such as Hel2p $[9,11,16,47]$. However, in the disome, the interface between the two ribosomes was relatively flexible; the contacts between the two ribosomes were much weaker or nearly absent in our structure of the disome (Fig. 6c, Additional file 1: Fig. S6b-h). For instance, a tight interaction was reported between the two RACK1 (Asc1p) - the protein involved in the initiation of RQC-from the two ribosomes in the di-ribosome [9]. However, the interaction was completely lost in the disome (Fig. 6d, e), due to the different orientation of the two $40 \mathrm{~S}$ subunits (Fig. 6b). These observations suggest that the disome may lack specific structural features required for recruiting RQC factors, such as Hel2p.

Second, the leading ribosome of the majority $(31,010 / 35,918=86.3 \%)$ of disomes was detected in the rotated state (Fig. 6a), in sharp contrast to the non-rotated state (with 


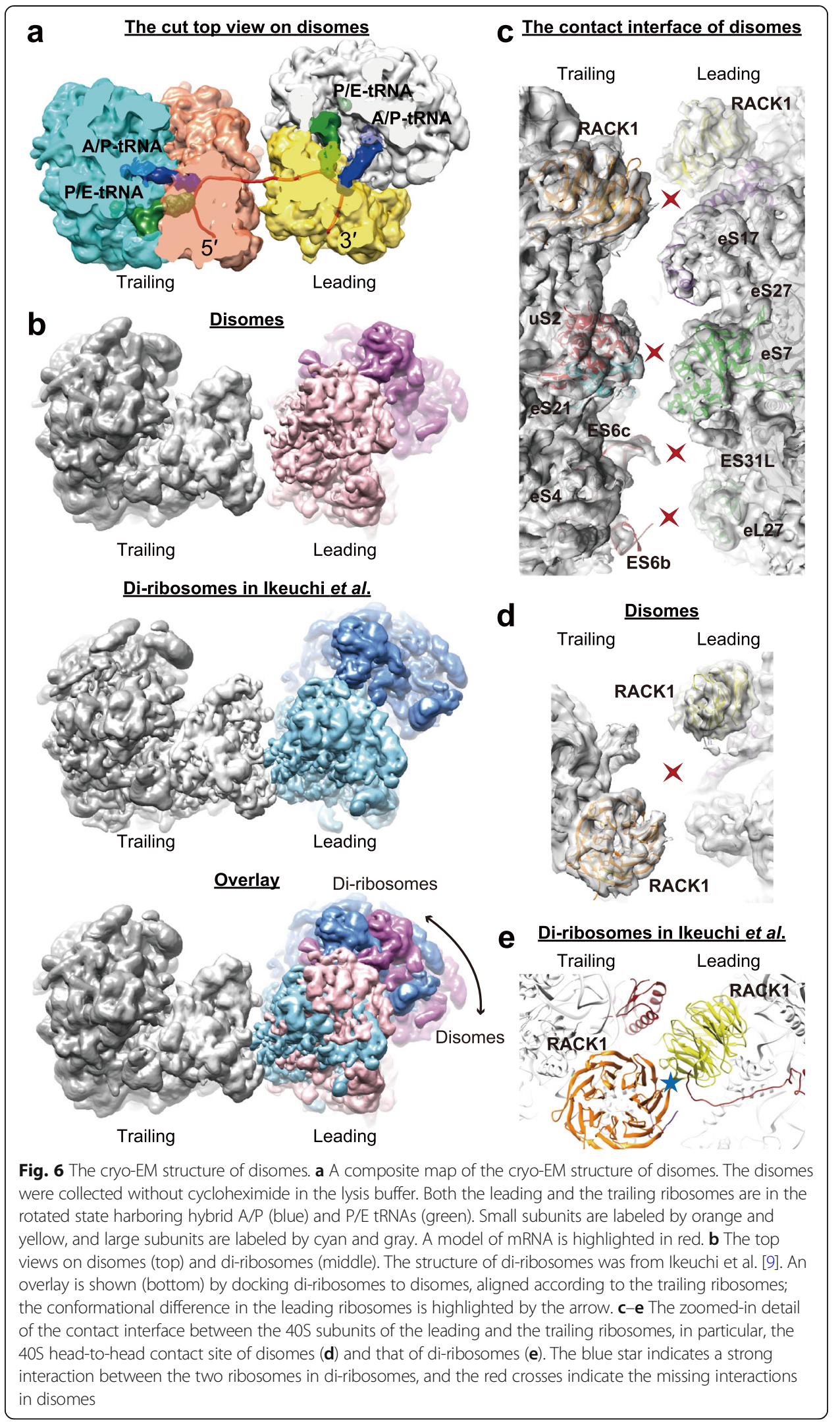


$\mathrm{P} / \mathrm{P}$ and $\mathrm{E} / \mathrm{E}$ tRNAs) reported for the leading ribosome in di-ribosomes. The nonrotated state has been previously reported as a feature of stalled $80 \mathrm{~S}$ ribosomes before collisions [9], and therefore, the rotated state of the leading ribosome indicates that these disomes are likely induced by transient pauses. Collectively, the results of cryo-EM analyses indicate that the widespread disomes observed in vivo (Fig. 2c) are structurally different from di-ribosomes that are recognized and resolved by the RQC pathway.

\section{Ribosomes are associated with specific chaperones}

To understand the function of endogenous ribosome collisions, we attempted to identify disome-specific ribosome components. We labeled ribosome proteins with stable isotopes, digested the mRNA with RNase I, and separated disomes from monosomes with sucrose density gradient centrifugation. We mixed an equal amount of protein extracted from the heavy-labeled disome fraction with light-labeled monosome fraction (or light-labeled disome fraction with heavy-labeled monosome fraction in a label-swap replicate) and performed tandem mass spectrometry (MS/MS, Fig. 7a).

In addition to ribosomal proteins, some highly expressed metabolic enzymes were also identified (Additional file 1: Fig. S7, and Table S4, S5); for two reasons, we speculate that they are nascent peptides of incomplete protein translation attached to the ribosomes. First, the peptides of these enzymes captured by MS/MS tended to be in the first half of the coding sequences (the common odds ratio $=1.9, P=0.001$, the MantelHaenszel test). For example, all detected peptides of FAS2p, a fatty acid synthetase, were in the first half (Additional file 1: Fig. S7b). By contrast, the peptides of ribosomal proteins captured by MS/MS were evenly distributed in the first and second half of the coding sequences (the common odds ratio $=1.0, P=0.7$ ). Second, among the 26 metabolic enzymes, the protein abundance of a metabolic enzyme in the disome fraction was positively correlated with the abundance of disome footprints on its encoding gene ( $\rho=0.57, P=2.5 \times 10^{-3}, N=26$, Spearman's correlation, Additional file 1: Fig. S7c). By contrast, we did not detect such correlation for ribosomal proteins $(\rho=0.16, P=0.08$, $N=129$, Spearman's correlation, Additional file 1: Fig. S7c); this is not unexpected since most ribosomal proteins were likely captured by MS/MS as ribosome components instead of as nascent peptides.

Most proteins have similar abundance per ribosome in disomes and in monosomes (e.g., two copies in a disome and one copy in a monosome) and therefore were on the diagonal in the scatter plot showing protein intensities in disomes vs. in monosomes; many were constitutive ribosome components (Fig. 7b and Additional file 1: Fig. S7a). Proteins above the diagonal line were the disome-enriched proteins (Fig. 7b, Additional file 1: Fig. S7a, and Table S4, S5). Proteins involved in the RQC pathway such as Hel2p, Slh1p, Cue3p, and Rqt4p [9, 16, 48] were not observed significantly enriched in disomes (Additional file 1: Table S4, S5).

In contrast, all 11 chaperones identified by our MS/MS analysis had $>1.5$-fold perribosome abundance in disomes than in monosomes $\left(P=1 \times 10^{-6}\right.$, Fisher's exact test, Fig. 7b; $P=1 \times 10^{-4}$, Additional file 1: Fig. S7a). For example, although SSB1p was reported as a ribosome-associated chaperone [49], how SSB1p is distributed among ribosomes remained unclear. The MS/MS analyses indicate that SSB1p is associated 


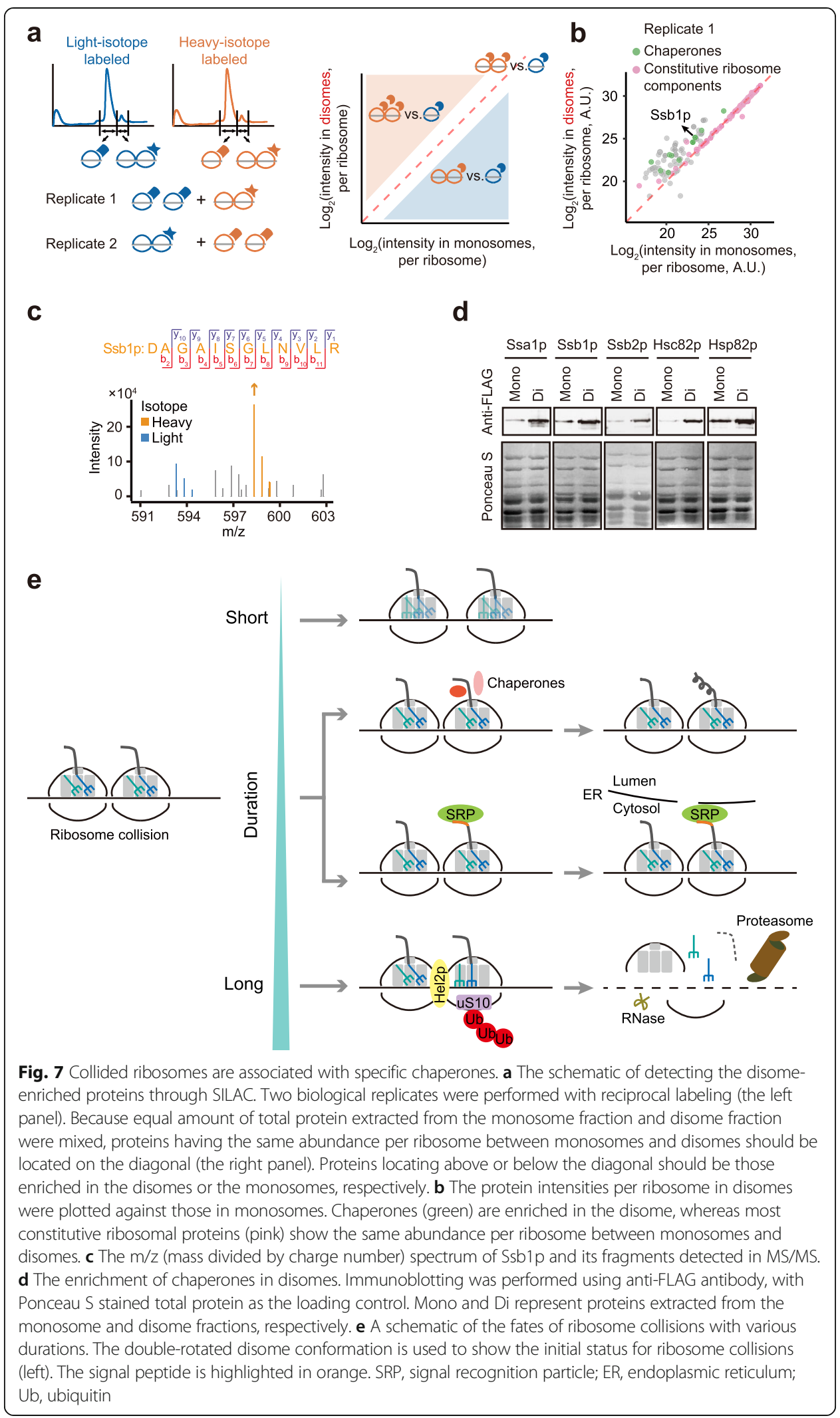


primarily with disomes (Fig. 7b, c). To validate this result, we randomly chose five out of these 11 chaperones and fused $4 \times$ FLAG to the C-terminus for each of them. The enrichment in disomes was confirmed with the immunoblotting assay for all five chaperones (Fig. 7d). These observations suggest that some chaperones are associated with ribosome collisions, capable of participating in the folding of nascent peptides.

\section{Discussion}

\section{Caveats}

There are at least four caveats in this study since we assumed that the abundance of disome footprints faithfully reflects the intensity of ribosome collisions at a genomic location. First, if there is any relation between disome footprints and the transcripts binding to two ribosomes due to incomplete RNase I digestion, disome footprints may not always indicate ribosome collisions. To test this possibility experimentally, we collected the transcripts binding to two ribosomes, performed RNase I digestion, and sequenced ribosome-protected mRNA fragments (Additional file 1: Fig. S8a). The footprints repeatedly observed in disome-seq did not show up in such experiments (Additional file 1: Fig. S8b as an example), indicating that disome footprints are fundamentally different from the transcripts binding to two ribosomes, in which the two ribosomes are likely sparsely distributed, and the mRNA fragment between them is sensitive to RNase I.

The second confounding factor is ligation bias. RNA fragments with various $5^{\prime}$-nucleotide compositions may have variable ligation efficiency with a fixed 5 '-adaptor sequence during the preparation of the high-throughput sequencing libraries [50,51]. To reduce such ligation bias, we had added three random nucleotides to the 3 '-end of the 5 '-adaptor during library preparation. To assess how much ligation bias is remained, we calculated the frequency for each of the sixty-four 3-mers in the -15 th to -13 th nt region upstream of the A-site of monosome footprints, as well as in the -45 th to -43 th nt region upstream of the A-sites of the leading ribosomes of disome footprints. Both were positively correlated with the genome-wide tri-nucleotide composition in the coding sequences (Additional file 1: Fig. S9a-c), indicating that ligation bias has been largely eliminated from our monosome-seq and disome-seq libraries.

Nevertheless, we realized that any ligation bias not entirely eliminated could have led to discrepancies in the translational pauses detected by disome-seq and monosome-seq. It is because a translational pause generates different 5 '-end for monosome and disome footprints (Additional file 1: Fig. S9a). To assess such possibility, we estimated the 3mer frequencies in the -15 th to -13 th nt region upstream of the A-sites (for disomes, the A-site of the leading ribosome) for translational pauses detected in disome-seq or monosome-seq, respectively. Such 3-mers are the 5 '-end of the 28-nt monosome footprints but are the internal sequences of disome footprints. Although monosome-seq and disome-seq identify largely non-overlapping pausing sites (Fig. 2e), the 3-mer frequency in the -15 th to -13 th nt region upstream of the A-sites were positively correlated between the two experiments $\left(\rho=0.95, P<2.2 \times 10^{-16}, N=64\right.$, Spearman's correlation, Additional file 1: Fig. S9d). This observation indicates that ligation bias cannot be a major explanation for the discrepancy in the locations of translational pauses identified by disome-seq or monosome-seq. 
A third caveat is related to the background used in the Mantel-Haenszel test for calculating the pausing scores. We performed appendant mRNA-seq experiments that mRNA was sheared into <50-nt fragments using a high-salt buffer, instead of the RNase I digestion as in the monosome-seq and disome-seq experiments. The purpose of using such mRNA-seq data as the background in our analyses (Fig. 3, 4, 5) was to control for any potential ligation bias, amplification bias, or sequencing bias in the high-throughput sequencing. Nevertheless, we realized that another approach was to use codon or amino-acid frequencies in each gene as the background (Additional file 1: Fig. S10a). We recalculated the A-site and P-site pausing scores using this approach and found them virtually unchanged (Additional file 1: Fig. S10b, c), indicating that the reported pausing scores in this study are computationally robust.

Last, it is worth noting that the ribo-seq protocol has been "evolving" since its debut in 2009 [17]. In the initial protocol, yeast cells were co-cultured with cycloheximide, a step that was later shown distorting the location of ribosome footprints within genes $[19,20$, 52]. Furthermore, the addition of cycloheximide, as well as other antibiotics, in the lysis buffer will stabilize ribosomes at various conformations that protect mRNA fragments of different sizes [51, 53]. The size selection for ribosome footprints has also been shown to have unexpected effects on the identification of translational pausing sites [54]. We avoided co-culturing yeast cells with cycloheximide but added cycloheximide in the lysis buffer to stabilize ribosomes, following recently updated ribo-seq protocols $[19,20,53$, 55]. Nevertheless, it is reasonable to assume that although disome-seq represents some advance in understanding ribosome collisions, it inevitably reflects only part of the whole story, due to technical limitations of the experimental procedures.

Similarly, it remains unclear how the chemicals in the lysis buffer could have affected the structural determination of disomes by cryo-EM. In addition to the disomes collected with cycloheximide omitted from the lysate (Fig. 6, Additional file 1: Fig. S6), we also determined the structure of the disomes extracted with cycloheximide added to the lysis buffer (as in the disome-seq experiment). These disomes held the two structural features we reported in Fig. 6: a markedly different interface from the di-ribosome and a rotated state for the leading ribosome (Additional file 1: Fig. S11a-c). Nevertheless, the structural determinations of disomes under various experimental conditions are recommended in the future for a more comprehensive understanding of ribosome collisions.

\section{The 61-nt disome footprints}

Although not highlighted in our results, note that we did conduct additional analyses to examine the length distribution of disome footprints. In addition to the 58-nt disome footprints whose conformation was determined in Fig. 1e as two 28-nt monosome footprints spaced by two nts, the $5^{\prime}$-end of the 61-nt fragments also exhibited an apparent 3-nt periodicity (Fig. 1d, 2b), suggesting the presence of a second disome conformation. The disomes accumulated at the stop codons (Additional file 1: Fig. S12a) indicate that the 61-nt disome footprints were composed of two 28-nt monosome footprints spaced by five nts. The fate of such 61-nt disomes is likely determined by which of the two events occurs first. If the leading ribosome resumes translation first, the ribosome collision is resolved; alternatively, if the trailing ribosome moves first, probably because the leading ribosome has paused for a long time, a 58-nt disome is formed. 
The 61-nt disome footprints were more abundant than the 58-nt disome footprints in cells growing in the rich medium (Fig. 2b, Additional file 1: Fig. S13a). The abundance of the 61-nt disome footprints was reduced when cells were treated with 3-AT (Fig. 1d, Additional file 1: Fig. S13a), a phenomenon that was also observed in a recent study [56]. We speculate that the translational pauses at the histidine codons induced by the 3-AT treatment last longer than the time frame of an average translational pause for yeast cells growing in the rich medium, thereby providing additional time for one more move of the trailing ribosome toward the leading ribosome.

To determine if the distinction between the 58-nt and the 61-nt disome footprints can provide additional information for the strength of a ribosome collision, we separately estimated the pausing scores from the 58-nt or the 61-nt disome footprints. They were highly correlated (for the A-site, $\rho=0.92, P<2.2 \times 10^{-16}, N=64$; for the P-site, $\rho=0.88, P=3.2 \times 10^{-7}, N=20$, Spearman's correlations, Additional file 1: Fig. S12b), indicating the absence of length-specific collision signals between the 58-nt and the 61-nt disome footprints. To improve the statistical power, we combined the 58 and 61-nt footprints for the analyses in Fig. 3, 4, 5. A recent investigation on mouse liver also reported two lengths of disome footprints, 59-60 nts and 62-63 nts; similar to our observations, nearly identical pausing sequences were reported for the disome footprints of these two lengths [57].

\section{The 53-nt disome footprints}

In addition to the 58-nt and the 61-nt disome footprints, we observed the 53-nt disome footprints, which showed an apparent 3-nt periodicity (Fig. 1d, 2b) and accounted for $10.9 \%$ of disome footprints for yeast cells growing in the rich medium (Additional file 1: Fig. S13a). This indicates the existence of a third disome conformation. When treated with 3-AT, the 5'-end of most 53-nt disome footprints was 45-nt upstream of the histidine codons (Additional file 1: Fig. S13b), the same position as in the 58-nt disome footprints (Fig. 1e). It indicates that the positions of the A-site of the leading ribosome relative to the 5 '-end are identical between the 53-nt and the 58-nt footprints. This observation echoes the two lengths of monosome footprints reported in previous studies, $\sim 21 \mathrm{nts}$ and $\sim 28 \mathrm{nts}$ [51, 53]; these two footprints share the same 5 '-end but show different 3 '-end. The 28-nt footprints represent the ribosomes whose A-sites are occupied (in the rotated state with hybrid $\mathrm{A} / \mathrm{P}$ and $\mathrm{P} / \mathrm{E}$ tRNAs or the non-rotated state with $\mathrm{A} / \mathrm{A}$ and $\mathrm{P} / \mathrm{P}$ tRNAs); the length of footprints reduces to $\sim 21 \mathrm{nts}$ when the A-site of a ribosome is open (in the non-rotated state with $\mathrm{P} / \mathrm{P}$ and E/E tRNAs) [53].

In alignment with the two lengths of monosome footprints, we speculate that the $(28+2+23=) 53$-nt disome footprints represent a disome conformation that the A-site of the leading ribosome is open, whereas the $(28+2+28=) 58$-nt disome footprints represent a conformation that the A-site of the leading ribosome is occupied. If true, we predict that the abundance of the 53-nt disome footprints will increase when cells are treated with 3-AT; it is because 3-AT induces ribosome collisions mainly through a prolonged time frame for decoding histidine codons, the very state that the A-site of the leading ribosome is open. The proportions of the 53-nt footprints indeed increased from $10.9 \%$ for cells growing in the rich medium to $17.9 \%$ for cells treated with 3 -AT 
(Fig. 1d, 2b, Additional file 1: Fig. S13a), in support of our interpretation of the 53-nt disome footprints.

If the 53-nt disome footprints represent a disome conformation that the A-site of the leading ribosome is open, we further predict the existence of disome structure that the leading ribosome is in the non-rotated state with $\mathrm{P} / \mathrm{P}$ and $\mathrm{E} / \mathrm{E}$ tRNAs (presumably protecting 23-nt mRNA fragment) and the trailing ribosome is in the rotated state with hybrid A/P and P/E tRNAs (protecting 28-nt mRNA fragment). Indeed, we observed that such conformation in $(4908 / 35,918=) 13.7 \%$ of the disomes collected from yeast cells growing in the rich medium (Additional file 1: Fig. S11d). Nevertheless, the specific contacts between the two $40 \mathrm{~S}$ subunits observed in di-ribosomes $[9,16]$ remained absent in these disomes (Additional file 1: Fig. S11e).

For cells growing in the rich medium, the stop codons were less representative in the A-site of leading ribosomes in the 53-nt disome footprints (Additional file 1: Fig. S13c, d), echoing the 52-nt and 54-nt disome footprints recently reported in human cells [38]. In the study, the 52-nt and 54-nt footprints were protected by the disomes that the A-site of the leading ribosome was open and tended to locate in coding sequences compared to the stop codon. On the other hand, the 58 and 61-nt disome footprints detected in our study are similar to the $\sim 61$-nt footprints reported in human cells; they showed a stronger tendency to locate at stop codons (Fig. 3e, Additional file 1: Fig. S13c) [38]. The reduced enrichment of the 53-nt disome footprints at stop codons (Fig. 3e, Additional file 1: Fig. S13c, d) suggests that stop codons are rapidly occupied by release factors in the A-site of ribosomes; the translation is slow at stop codons probably because of the prolonged subsequent steps disassembling the posttermination ribosomal complexes [58].

If the 53-nt and the 58-nt/61-nt footprints are protected by disomes that the Asite of the leading ribosome is open and occupied, respectively, we reasoned that the 58-nt/61-nt disome footprints should be more informative than the 53-nt ones in detecting the translational pauses associated with peptide bond formation and translocation. It is because these processes slow down translation elongation only if the A-site of a ribosome is occupied. Indeed, when calculated from the 53-nt disome footprints, the P-site pausing score of proline, the amino acid that is well known as a poor acceptor for peptide bond formation [40, 46], was no longer significantly $>1$; such pausing signal was observed for the 58-nt disome footprints (Fig. 4a, Additional file 1: Fig. S13e).

We further reasoned that the 53-nt disome footprints should be more informative than the 58-nt ones in detecting the effect of codon optimality, because codon optimality causes translational pauses only if the A-site of a ribosome is open [53]. However, we did not observe more ribosome collisions associated with non-optimal codons than optimal codons using either the 53-nt disome footprints ( $y$-axis in Additional file 1: Fig. S13c, $P=0.47, N=61$, Mann-Whitney $U$ test) or the 58-nt/61-nt disome footprints $(x-$ axis in Additional file 1: Fig. S13c, $P=0.85, N=61$, Mann-Whitney $U$ test). In fact, the A-site pausing score for the 61 amino-acid codons was highly correlated between the 53-nt and the 58-nt/61-nt disome footprints (Additional file 1: Fig. S13c, $\rho=0.56, P=$ $3.5 \times 10^{-6}, N=61$, Spearman's correlation). Our observation is consistent with Han et al., who reported in human cells that synonymous codon usage/tRNA supply was poorly associated with the propensity for ribosome collisions [38]. 
Synonymous codons are known to be recognized at variable rates [19, 20, 53], which further regulate mRNA stability [31, 59]; however, the impact of codon optimality on ribosome collisions was not detected in disome-seq. One explanation is that decoding is a relatively rapid step in the work cycle of translation elongation [60]. Therefore, its potential in inducing variable ribosome collisions is masked by variation in the duration of other steps such as peptide bond formation. Consistently, when the decoding time became a dominant factor in the work cycle of translation elongation as the yeast cells were treated with 3-AT, the non-optimal codon of histidine, CAT, did induce more ribosome collisions than the optimal codon, CAC (Fig. 1e, Additional file 1: Fig. S13b, f).

\section{The evidence for the existence of trisomes}

In addition to disomes, translational pauses may further lead to longer queues of collided ribosomes, for example the collision of three ribosomes-trisomes [15, 56, 61]. Indeed, after RNase I digestion, a small number of particles were observed at the $\sim 62$ $\mathrm{mm}$ position after sucrose gradient centrifugation in Fig. 1b; the density of these particles was higher than disomes and was similar to transcripts binding to the three ribosomes, suggesting that they were trisomes. Although the abundance of these trisomes was insufficient for a trisome-seq experiment, the presence of trisomes upstream of stop codons was evident by the disome footprints with the $5^{\prime}$-end $\sim 75$-nt upstream of the stop codon (Additional file 1: Fig. S12c).

\section{The absence of translational "ramp"}

Yeast genes often use more non-optimal codons in the $5^{\prime}$ region of the coding sequences, which has been speculated to serve as a translational "ramp" to reduce downstream ribosome collisions [62]. However, we did not find the signals that non-optimal codons induced more ribosome collision (Fig. 3d, Additional file 1: Fig. S13c) nor did we detect any accumulation of ribosomes downstream of the start codon in either monosome-seq or disome-seq (Fig. 3e). It is likely that the previously observed accumulation of ribosomes around the start codon [17] is a byproduct of the co-culturing with cycloheximide $[19,20,61]$ that partially inhibits translation elongation but does not block initiation [63].

Instead, we observed more ribosome collisions downstream of a transcript (Fig. 3e), an "inverse ramp" that in our view can be partly explained by two mutually nonexclusive mechanisms. First, ribosomes tend not to collide in the upstream region of an mRNA due to a steric effect. The small subunit occupies $\sim 37$ nts of mRNA during subunit joining, with 22 nts downstream of the start codon [64]. This conformation requires the 5 '-end of the leading ribosome at least 24-nt downstream of the start codon; when translation elongation starts, the start codon is at the P-site of the ribosome, indicating that such ribosomes occupy 13 nts downstream of start codons (Additional file 1: Fig. S14a). Therefore, such a steric effect sets a minimum 11-nt distance between two ribosomes at translation initiation, reducing the possibility of ribosome collisions in the $5^{\prime}$-region of coding sequences. For example, even if the leading ribosome stays still, it requires three moves of the trailing ribosome to collide (Additional file 1: Fig. S14a). Consistent with this model, disomes were missing entirely in the first nine nts of the open reading frame of any gene (Additional file 1: Fig. S14b). The collisions propensity 
increases gradually along the coding sequence, reaching a plateau $\sim 50$ nts after translation starts, as indicated by the meta-gene analysis of the disome-seq data (Additional file 1: Fig. S14b).

A second explanation is that translation termination is relatively slow compared to elongation, resulting in ribosomal "traffic jam" upstream of stop codons; abundant disomes (Fig. 3e) and even some trisomes were formed close to stop codons (Additional file 1: Fig. S12c). It can be reasonably generalized that the elevated ribosome density due to slow termination can result in more frequent ribosome collisions in a region longer than the $\sim 90 \mathrm{nts}$ that are occupied by trisomes.

Note that both steric effects aforementioned, ribosomes lining up equidistantly downstream of start codons and queueing upstream of stop codons for translation termination and recycling, should have greater impacts on shorter genes, for two reasons. First, ribosomal flux is generally higher in shorter genes [33], amplifying both steric effects. Second, the same affected genomic region (e.g., a 90-nt region occupied by trisomes) will take up a larger fraction of the coding sequence in shorter genes. Consistently, the inverse ramp appears to be steeper among genes with shorter coding sequences (Additional file 1: Fig. S14c).

\section{Additional causes of ribosome collisions}

In addition to the sequence features identified at the A-site, the P-site, and the exit tunnel (Fig. 3, 4, 5), sequences outside of the ribosome may also render heterogeneity in the elongation rate. For example, mRNA secondary structure has been shown to cause translational pausing [23]. mRNA downstream of disome footprints exhibited a stronger secondary structure (Additional file 1: Fig. S15), indicating its role in inducing ribosome collisions. Relatedly, RNA helicases TIF1p and TIF2p were associated with disomes (Additional file 1: Table S4, S5), potentially removing mRNA secondary structures to relieve translational pauses. There are also sequence features that lead to ribosome collisions with unknown mechanisms. For example, two collision-inducing 3mers, QQQ and GGG, were identified in the exit tunnel (Fig. 5b); they are not positively charged. Nevertheless, both are related to protein folding to some extent. The former is a well-known signal for protein misfolding [65], and the latter is a prominent signal for intrinsic protein disorder [66].

\section{The benefit from ribosome-collision mediated cotranslational protein folding}

It is apparently costly when ribosomes are sequestered upstream of the stop codon, waiting for release, since they are not used for active protein synthesis. Nevertheless, slow ribosome release may benefit the cell by providing the newly synthesized peptide sufficient time to fold within the exit tunnel of ribosomes [67], rather than in the complex cytoplasmic environment. Some amino-acid sequences (e.g., proline and glycine at the P-site as well as polylysine in the exit tunnel) induce translational pauses as well (Figs. 4 and 5); they are also signals for the translation completion of $\alpha$-helices (Fig. 4c, d). These amino-acid sequences may provide the time and subcellular environment for the folding of newly synthesized peptides inside the exit tunnel, especially $\alpha$-helices. The narrowest region of the exit tunnel, the constriction site, is $\sim 10 \AA$ in width [68], sufficient for cotranslational folded $\alpha$-helices ( $\sim 5 \AA$ in width) [69] to pass by. It would 
be important in the future to test if the manipulation of the translational pausing signals can affect protein folding.

Such a dual role of amino acids can catalyze the evolution of protein structure because it does not take additional time for placing a translational pausing signal after an evolutionary change in protein structure-the amino-acid substitution that results in innovation in protein structure at the same time confers a translational pause, making the emergent protein structure ready to be folded. Together with the cotranslational chaperones associated with collided ribosomes, the proper folding of a novel protein structure is warranted during evolution.

\section{The fates of ribosome collisions}

The key to studying ribosome collision-mediated translational regulation is to unveil the dynamics of ribosomes during translation. Traditional ribo-seq provides the location information of monosomes but misses collided ribosomes, which are present in $46 \%$ of genes in yeast cells growing in the rich medium and provide unique information about translational pausing. Three similar studies in humans and zebrafish [38], yeast [56], and mice [57] were recently published during the preparation of our manuscript. All four studies, including our own, performed disome-seq and showed that ribosome collisions were widespread, despite the use of various model organisms.

We performed structural analyses on yeast disomes; 86.3\% disomes were composed of two ribosomes that both were at the rotated state (Fig. 6a). This structure is consistent with the $(28+2+28=) 58$-nt or the $(28+5+28=) 61$-nt disome footprints that the same length of mRNA (28 nts) was protected by the two ribosomes. By contrast, when the leading ribosomes switch to the non-rotated state with P/P and E/E tRNAs, the two ribosomes will protect different lengths of mRNA (28 and 23 nts for the trailing and leading ribosomes, respectively). In mouse liver, Arpat et al. reported that the majority of disomes protected $(29+1+29=) 59 \mathrm{nts}$ or $(29+4+29=) 62 \mathrm{nts}$ [57]. Since the same length of mRNA ( $29 \mathrm{nts}$ ) was also protected by the two ribosomes, the lengths of these disome footprints can be explained by the double-rotated disome conformation determined in this study.

The data collected in the four studies led us to propose an integrated model for the ribosome-collision mediated translational regulation. Since an mRNA is translated simultaneously by multiple ribosomes, ribosome collisions are likely inevitable. The high ribosomal flux on an mRNA will increase the chance of collisions in general (Fig. 2d) due to the inherent stochasticity of biochemical reactions, on the basis of which sequence-associated heterogeneity in the elongation rate further promotes the propensity of collisions at specific locations. Besides, some ribosome collisions may be induced by aberrant transcripts or arrested nascent peptides.

There are at least three possible fates for these ribosome collisions observed in this study. Firstly, some ribosome collisions are likely transient, and the translation of both ribosomes can be resumed (Fig. 7e, top path); this could be the mechanism that keeps the ribosome density largely constant over a transcript (Fig. 3e, bottom panel, Additional file 1: Fig. S14c) in spite of widespread collided ribosomes $(\sim 5.8 \%$ at the moment that the disome "snapshot" was taken, Fig. 1b; or $\sim 10 \%$ estimated in mouse liver [57]). Second, some ribosome collisions may last for a more extended period; these 
collisions may promote cotranslational protein folding or aid in targeting to correct subcellular locations $[57,70]$ (Fig. 7e, middle path). Third, if ribosome collisions last for a sufficiently long time, the leading ribosomes will shift to the non-rotated stalling state, and a more rigid interface between the collided ribosomes will trigger the recognition by the RQC pathway, leading to the degradation of mRNA and nascent peptides $[9,15$, 16, 38, 56] (Fig. 7e, bottom path).

It is not yet clear the determinants of the collision duration, the mechanisms by which cells can sense how long a ribosome collision has persisted, and the fraction of ribosome collisions destined for each of the three fates. It is also unclear the mechanisms by which cells distinguish programmed (for protein folding or subcellular localization) and sporadic collisions (due to aberrant transcripts and defective nascent peptides). Considering the central role of ribosome collisions in protein homeostasis, these are key issues that need to be studied in the future.

\section{Conclusions}

Ribosome collisions are widespread in fast proliferating yeast cells, especially on mRNA with high ribosomal flux. Ribosome collisions tend to occur at stop codons and are often related to the translation completion of $\alpha$-helices. A large number of collided ribosomes are structurally incompetent to trigger the RQC pathway; instead, they are often associated with chaperones, which likely aid in cotranslational protein folding. Taken together, we offer a mechanism that chaperones sense translation elongation rate through ribosome collisions to determine which proteins/peptide regions require cotranslational folding.

\section{Methods}

Polysome profiling and disome-seq

\section{Sample preparation}

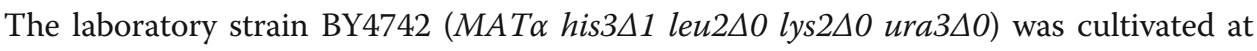
$30{ }^{\circ} \mathrm{C}$ in the rich medium YPD (1\% yeast extract, $2 \%$ peptone, and $2 \%$ dextrose). For experiments of the 3-AT treatment, strain $\mathrm{S} 288 \mathrm{C}$ was cultivated at $30{ }^{\circ} \mathrm{C}$ in the $\mathrm{SC}-\mathrm{His}+$ 3-AT medium (synthetic complete medium with histidine dropped-out and $100 \mathrm{mM} 3-$ AT added). We harvested cells following previous studies [24]. Briefly, cells were cultured for over two doubling cycles in the absence of cycloheximide, were collected at $\mathrm{OD}_{660} \sim 0.6$ by vacuum filtration, and were immediately frozen in liquid nitrogen. Ribosomes were extracted with the polysome lysis buffer (PLB), which contained $200 \mathrm{mM}$ Tris- $\mathrm{HCl}$ (pH 8.0), $200 \mathrm{mM} \mathrm{KCl,} 35 \mathrm{mM} \mathrm{MgCl}$, 1\% (v/v) Triton X-100, 5 mM DTT, and $50 \mu \mathrm{g} / \mathrm{mL}$ cycloheximide.

\section{Polysome profiling}

The extracted ribosomes were pelleted through a $30 \mathrm{~mL}$ sucrose cushion containing $400 \mathrm{mM}$ Tris- $\mathrm{HCl}$ (pH 8.0), $200 \mathrm{mM} \mathrm{KCl,} 30 \mathrm{mM} \mathrm{MgCl}$, $1.75 \mathrm{M}$ sucrose, $5 \mathrm{mM} \mathrm{DTT}$, and $50 \mu \mathrm{g} / \mathrm{mL}$ cycloheximide, by ultracentrifugation at $4{ }^{\circ} \mathrm{C}$ overnight $(33,500 \mathrm{rpm}$, Beckman, 70Ti rotor). The ribosome pellet was dissolved in $300 \mu \mathrm{L}$ RNase I buffer (20 $\mathrm{mM}$ Tris- $\mathrm{HCl} \mathrm{pH}$ 8.0, $140 \mathrm{mM} \mathrm{KCl,} 5 \mathrm{mM} \mathrm{MgCl}, 50 \mu \mathrm{g} / \mathrm{mL}$ cycloheximide, and $50 \mu \mathrm{g} /$ $\mathrm{mL}$ chloramphenicol) and separated by ultracentrifugation at $4{ }^{\circ} \mathrm{C}$ for $3 \mathrm{~h}(35,300 \mathrm{rpm}$, 
Beckman, SW41 rotor) through a 5-50\% sucrose gradient ( $40 \mathrm{mM}$ Tris- $\mathrm{HCl} \mathrm{pH} \mathrm{8.4,} 20$ $\mathrm{mM} \mathrm{KCl}, 10 \mathrm{mM} \mathrm{MgCl}_{2}$, and $50 \mu \mathrm{g} / \mathrm{mL}$ cycloheximide) prepared by Gradient Master (Biocomp). The profiling signals were recorded by Piston Gradient Fractionator (Biocomp).

\section{Monosome-seq, disome-seq, and mRNA-seq}

Libraries were prepared as described previously $[17,24,55]$, with modifications. Fifty thousand units $\left(\mathrm{A}_{260}\right)$ of ribosome dissolved in the PLB buffer were treated with $750 \mathrm{U}$ RNase I (Ambion, AM2294) at $25^{\circ} \mathrm{C}$ for $2 \mathrm{~h}$. Our pilot experiments, as well as previous studies, have shown that sucrose gradient centrifugation is not necessary $[55,71-73]$ as long as the digestion with RNase I is complete (Additional file 1: Fig. S1); rather, this step consumes a large amount of ribosome (especially disome) samples. The following-up computational analyses can help to tell if the RNA fragments being collected are largely protected by ribosomes: whether the fragments are restricted to the coding sequences, whether the fragments are enriched in certain lengths, and whether a 3-nt periodicity exists (Additional file 1: Fig. S3c-f). Therefore, RNA was directly extracted from the solution for RNase I digestion with hot phenol and was separated on a $17 \%(w / v) 7 \mathrm{M}$ urea denaturing polyacrylamide gel in a $0.5 \times$ Tris-borate-EDTA (TBE) electrophoresis buffer. RNA fragments with the length of approximate $20-30$ nts or $50-80$ nts were extracted by gel crushing and further incubated with an RNA gel extraction buffer (300 mM NaOAc pH 5.2, 10 mM Tris-HCl pH 8.0, 1 mM EDTA pH 8.0) overnight.

To control for technical bias during library preparation, appendant mRNA-seq was performed [17]. Specifically, total RNA was extracted using hot phenol, and $75 \mu \mathrm{g}$ extracted RNA was applied to mRNA purification using the Dynabeads ${ }^{\mathrm{ma}}$ mRNA purification kit (Life Technologies, 61006). For fragmentation, $11.1 \mu \mathrm{L} 10 \times$ fragmentation

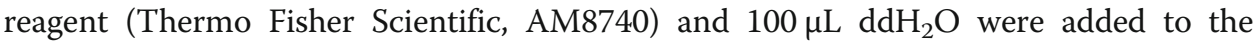
purified mRNA. Fragmentation proceeded for $30 \mathrm{~min}$ at $70^{\circ} \mathrm{C}$ and was aborted when $11.1 \mu \mathrm{L} 10 \times$ stop solution was added. Fragmented RNA was precipitated by isopropanol overnight and was separated on a $17 \%(\mathrm{w} / \mathrm{v}) 7 \mathrm{M}$ urea denaturing polyacrylamide gel. RNA fragments with the length $\sim 28 \mathrm{nts}$ were extracted from gel.

The extracted RNA fragments for monosome-seq, disomes-seq, or mRNA-seq were subjected to small RNA library construction for Illumina sequencing (Gnomegen, k02420). The 5'-RNA adaptor contained a 3-nt random sequence at the 3 '-end to avoid potential ligation bias. Monosome-seq/RNA-seq and disome-seq libraries were sequenced with single-end 50 and paired-end 100 modes on BGISEQ-500 (BGI Group), respectively.

\section{Mapping reads to the yeast genome}

The 3-nt random sequence at the $5^{\prime}$-end of each sequencing read was removed. The removed 3-nt sequence was added to the head of each read in the fastq format as the UMI, which can serve to remove PCR duplications generated during Illumina library preparation. The sequence identical to the 3 '-sequencing adaptor was also trimmed in each read using cutadapt V1.16 (http://gensoft.pasteur.fr/docs/cutadapt/1.6/index.html) [74]. Reads without the 3 '-adaptor sequence were removed since they ( $>46 \mathrm{nts}$ for monosome-seq or $>96 \mathrm{nts}$ for disome-seq) were much longer than the expectation 
(20-30 nts for monosome-seq and 50-80 nts for disome-seq). Trimmed reads shorter than 20 nts were also excluded from further analysis.

The Saccharomyces cerevisiae genome (SGD R64-1-1) (https://www.yeastgenome.org/ ) was used as the reference [75]. The trimmed reads were mapped against rRNA with bowtie V1.2.2 [76] (http://bowtie-bio.sourceforge.net/manual.shtml); the mapped reads were filtered to avoid rRNA contamination. The rest reads were aligned against coding sequences with the --no-novel-juncs parameter using Tophat V2.1.1 [77] (https://ccb. jhu.edu/software/tophat/manual.shtml). Reads with multiple alignments or with mapping quality $<30$ were discarded. Biological replicates were highly correlated (Additional file 1: Fig. S2) and were combined in the majority of our analyses. To remove PCR duplicates generated during Illumina library preparation, sequencing reads of the same length, sequence, and UMI were counted only once. The number of disome reads obtained in this study (Additional file 1: Table S2) are comparable to those in previous studies [24].

\section{The definition of the A-site}

The definition of A-site in the disome footprints with various length is overviewed in Additional file 1: Fig. S16. Specifically, for the 58-nt disome footprints, if the 5 '-end of a read was mapped to the first nucleotide of a codon (in frame), the 46th-48th nts was defined as the A-site of the leading ribosome; if mapped to $+1($ or +2$)$ frame, the 45th-47th (or 47th-49th) nts was defined as the A-site since the footprint likely shifted by +1 and -1 nt during RNase I digestion. The A-site of the leading ribosome was also defined for the 59-nt disome footprints: if the $5^{\prime}$-end was in frame, meaning that one more nt was kept at the $3^{\prime}$-end during RNase I digestion, the 46th-48th nts was the Asite. If mapped to +2 frame, meaning that one more nt was kept at the $5^{\prime}$-end during RNase I digestion, the 47th-49th nts was the A-site. For the 61 and 62-nt disome footprints, the A-site was defined assuming that an extra codon was included in the space between two ribosomes. The A-site of the 53 and 54-nt disome footprints was defined as that of the 58 and 59-nt disome footprints since the footprints were trimmed from the 3 '-end. For the 28 and 29-nt monosome footprints, the definition of the A-site was the same as the 58 and 59-nt disome footprints except for a 30-nt offset.

\section{The calculation of the pausing scores}

The A-site pausing score of each of the 64 codons was defined as the common odds ratio among genes calculated by the Mantel-Haenszel test. A $2 \times 2$ contingency table was generated for each gene. Disome footprints were divided into two categories, the concerning codon and other codons at the A-site of the leading ribosome. mRNA reads were used as the background to control for codon frequency in the gene; the "A-site" of a 28-29 nt mRNA-seq read was defined as if it was a 28-29 nt monosome footprint. The P-site pausing score was estimated similarly with the amino acid at the P-site under consideration. The exit-tunnel pausing score was estimated for each of the amino-acid 3-mers in the 20-amino-acid region upstream of the P-site; this length was conservative since peptides with 33 to 67 amino acids can be folded within the exit tunnel [78]. Flanking 3-mers may hitchhike on the causal 3-mer, and therefore, we 
combined multiple disome reads mapped to the same genomic position into one to reduce such a hitchhiking effect.

Statistical analyses were performed with $R$ (https://www.r-project.org/), and plots were generated with an $R$ package, ggplot2 (https:/ggplot2.tidyverse.org/). The $Q$ values were calculated using an $R$ package, qvalue (http://github.com/jdstorey/qvalue). All statistical tests were two-sided unless otherwise specified.

\section{Dual-luciferase assays}

The Renilla and firefly-luciferase sequences were obtained from the pTH727-CEN-RLuc/staCFLuc plasmid (http://www.addgene.org) and were concatenated with a linker sequence GGTCGACGGATCCCCGGG between them, for the purpose of keeping correct protein folding. This Renilla-linker-firefly fragment was further inserted into the p426 plasmid and was expressed under TDH3 promoter. The sequences of AAGAAGAAG, AAGAAGAAA, AAGAAAAAG, AAAAAGAAG, AAGAAAAAA, AAAAAGAAA, AAAAAAAAG, and AAAAAAAAA were individually inserted upstream of the coding sequence of firefly luciferase. Individual plasmids were transformed into BY4742. The plasmid-containing yeast clones were selected on SC-uracil plates.

The activity of luciferase was detected as described in a previous study [79]. Briefly, transgenic yeast strains were individually cultivated in the $\mathrm{SC}$-uracil medium $(150 \mu \mathrm{L})$ at $30^{\circ} \mathrm{C}$ overnight. Ten microliters of each yeast culture was transferred into a fresh SC-uracil medium $(140 \mu \mathrm{L})$ and was cultivated for another $4 \mathrm{~h}$ in a 96-well plate. Passive lysis buffer ( $40 \mu \mathrm{L}$, Promega, E1910) was added per well for cell lysis. Twenty-five microliters of the suspension was mixed with $25 \mu \mathrm{L}$ firefly luciferase substrate (Promega, E1910) and was incubated for $20 \mathrm{~min}$ at $25^{\circ} \mathrm{C}$. Firefly luciferase activity was measured by the Synergy multi-mode reader (BioTek). Twenty-five microliters of Stop-andGlo reagent (Promega, E1910) was added and mixed, which was incubated for another 20 min before the measurement of Renilla-luciferase activity.

\section{Cryo-EM}

\section{Disome purification}

Ten thousand units $\left(\mathrm{A}_{260}\right)$ of ribosome pellet were purified from cell lysates (cycloheximide omitted) and were subjected to digestion in $300 \mu \mathrm{L}$ RNase I buffer with $200 \mathrm{U}$ RNase I (Ambion, AM2294) at $25^{\circ} \mathrm{C}$ for $2 \mathrm{~h}$. The disome fraction was collected with sucrose gradient centrifugation and was concentrated through a $50-\mathrm{kDa}$ centrifugal filter (Millipore, UFC905096). The concentration was adjusted to $A_{260}=11.0$.

\section{Data acquisition}

Vitrified specimens were prepared by adding $4 \mu \mathrm{L}$ disome samples at the concentration of $\sim 150 \mathrm{nM}$ to a glow-discharged holey carbon grid (Quantifoil R1.2/1.3), which was covered with a freshly made thin carbon film. Grids were blotted for $1 \mathrm{~s}$ and plungefrozen into liquid ethane using an FEI Vitrobot Mark IV $\left(4{ }^{\circ} \mathrm{C}\right.$ and $100 \%$ humidity). The cryo-grids were initially screened at a nominal magnification of $\times 92,000$ in an FEI Talos Arctica microscope $(200 \mathrm{kV})$, equipped with an FEI CETA camera. High-quality grids were transferred to an FEI Titan Krios electron microscope operating at $300 \mathrm{kV}$, and images were collected using a K2 Summit direct electron detector (Gatan) in 
counting mode at a nominal magnification of $\times 105,000$, which corresponds to a pixel size of $1.356 \AA$ at the object scale (with defocus varying from -1.0 to $-2.0 \mu \mathrm{m}$ ). Movie stacks were collected semi-automatically using SerialEM [80]. Each micrograph was dose-fractionated to 32 frames with a dose rate of $\sim 10.0$ counts per physical pixel per second for a total exposure time of $6.4 \mathrm{~s}$.

\section{Data processing}

Original image stacks were summed and corrected for drift and beam-induced motion at the micrograph level using MotionCor2 [81]. SPIDER [82] was used for micrograph screening. The contrast transfer function parameters of each micrograph were estimated by Gctf [83]. All 2D and 3D classification and refinement were performed with RELION-3.0 [84].

The disome data were initially processed using $80 \mathrm{~S}$ monosome as a template for particle picking. A total of 1683 micrographs were collected, and 355,439 particles were picked for a cascade 2D and 3D classification with a binning factor of two. 281,953 particles with a box size of 400 pixels were split into 10 classes during the first round 3D classification (Additional file 1: Fig. S17).

Particles contained densities for two $80 \mathrm{~S}$ ribosomes were re-centered and reextracted with an enlarged box size of 440 pixels. After removing duplicated particles, 45,119 disome particles were subjected to another round of 3D classification. Two classes (a total of 35,918 particles) were pooled, and a soft mask on the leading ribosome was applied during the following mask-based 3D classification. Two groups were obtained: $86.3 \%$ of the particles contained $\mathrm{A} / \mathrm{P}$ and $\mathrm{P} / \mathrm{E}$ tRNA in the leading ribosome (group 1), the rest (13.7\%) containing $\mathrm{P} / \mathrm{P}$ and E/E tRNA in the leading ribosome (group 2). The 4908 particles in group 2 were subjected to 3D auto-refine with a soft mask on the trailing or leading ribosome, resulting in $7.96 \AA$ or $8.06 \AA$ maps, respectively. The 31,010 particles in group 1 were split into ten classes during the final round of 3D classification with a soft mask on the trailing ribosome. A total of 27,599 particles were subjected to $3 \mathrm{D}$ auto-refine with a soft mask on the trailing or leading ribosome, resulting in $4.85 \AA$ or $4.59 \AA$ maps (gold-standard FSC 0.143 criteria), respectively (Additional file 1: Fig. S17, S18).

\section{The structural determination of disomes collected in the cycloheximide-containing lysis buffer}

Disomes were also collected in the lysis buffer containing $50 \mu \mathrm{g} / \mathrm{mL}$ cycloheximide. The experimental and computational procedures were otherwise similarly performed. In brief, a total of 1439 micrographs were collected, and 269,962 particles were picked for a cascade 2D and 3D classification. A total of 4194 particles were applied for 3D autorefine with a soft mask on the trailing or leading ribosome, resulting in $7.11 \AA$ or 8.39 Å map, respectively.

\section{Mass spectrometry}

Stable isotope labeling with amino acids in cell culture (SILAC) was performed as described previously [85]. Briefly, a strain modified from BY4742 (MATa his $3 \Delta 1$ leu2 $\Delta 0$ lys $2 \Delta 0$ ura3 $\triangle 0$ arg $4 \Delta 0:: \mathrm{kanMX} 4$ car1 $\triangle 0:: L E U 2)$ was cultured at $30^{\circ} \mathrm{C}$ in the regular SC 
medium or in the SC medium substituted with heavy isotopes $(37.25 \mathrm{mg} / \mathrm{L}$ Lys8 and 20.94 mg/L Arg10). Lys8 and Arg10 represent ${ }^{15} \mathrm{~N}_{2}{ }^{13} \mathrm{C}_{6}$-lysine and ${ }^{15} \mathrm{~N}_{4}{ }^{13} \mathrm{C}_{6}$-arginine, respectively.

Cells were harvested at the mid-log phase $\left(\mathrm{OD}_{660} \sim 0.6\right)$, and polysomes were extracted. Polysome profiling was performed after RNase I digestion, and proteins were precipitated from the monosome or disome fraction with a double volume of 95\% ethanol, respectively. The protein precipitants were dissolved with the urea buffer ( $8 \mathrm{M}$ urea, $1 \mathrm{mM}$ sodium orthovanadate, $1 \mathrm{mM}$ sodium fluoride, $2.5 \mathrm{mM}$ sodium pyrophosphate, $1 \mathrm{mM}$ B-glycerophosphate, $0.2 \%$ tablet of protease inhibitors, and $1 \mathrm{mM}$ PMSF). One hundred fifty micrograms of light-labeled monosome protein was mixed with $150 \mu \mathrm{g}$ heavy-labeled disome protein; $150 \mu \mathrm{g}$ heavy-labeled monosome protein was mixed with $150 \mu \mathrm{g}$ light-labeled disome protein as a replicate. The protein mixtures were subject to the MS/MS analysis on Orbitrap Elite (Thermo Fisher Scientific).

The raw data were processed with MaxQuant V1.5.8.3 [86] (https://www.biochem. mpg.de/5111795/maxquant) using default parameters. All raw data were searched against the yeast proteome with the addition of potential contaminants. The protease was set as trypsin/P and trypsin. Two missed cuts were allowed. The protein intensities were retrieved from the output file (proteinGroups.txt) of MaxQuant. The proteins associated with ribosome collision can be identified by comparing the abundance per ribosome between disomes and monosomes. In principle, if a significant proportion of monosomes collected in the experiments were vacant, this comparison will identify proteins associated with translating ribosomes instead of those associated with disomes. However, this is an unlikely case since all 197,367 monosomes detected in our cryo-EM analyses accommodated tRNAs, indicating they are mostly translating ribosomes.

If the peptides of metabolic enzymes were detected in the ribosome particles because they were nascent peptides of incomplete protein translation, they should be enriched in the first half (N-terminus) of a protein. To determine if it is true, we divided the peptides detected into two categories, according to whether the $\mathrm{N}$-terminus of the peptide belonged to the first half of the protein or not. With the theoretical digested peptides (arginine or lysine at the C-terminus) as a background, a $2 \times 2$ contingency table was generated for each gene. The common odds ratio among genes was calculated by the Mantel-Haenszel test.

\section{Immunoblotting}

We did not have specific antibodies for the yeast chaperones enriched in disomes; therefore, we decided to perform immunoblotting for $4 \times$ FLAG tagged chaperones with the antibody against the FLAG tag. To this end, we constructed transgenic yeast lines where $4 \times$ FLAG tag was inserted to the C-terminus of five chaperones. Specifically, a DNA fragment composing the terminator of $A D H 1$ and the $\operatorname{kanMX}$ cassette was obtained from the pBS7 plasmid. $4 \times$ FLAG sequence was fused to the $5^{\prime}$-terminus of this fragment, which was further cloned into the pUC57 plasmid to generate the pUC57$4 \times F L A G-A D H 1$ terminator-kanMX plasmid (TSINGKE Biological Technology). Primers were designed to amplify this fragment and for recombination-based knock-in, right before the stop codon of each chaperone gene (Additional file 1: Table S6). 
Proteins were isolated from the monosome or disome fraction as described in the "Mass spectrometry" section. An equal amount of monosome and disome proteins were separated by $10 \%$ SDS-polyacrylamide gel and were transferred on to a Hybond ECL (GE Healthcare, RPN303D) membrane. The membrane was probed with antiDDDDK tag (binding to FLAG $^{\bullet}$ tag sequence, horseradish peroxidase-conjugated; Abcam, ab49763). Chemiluminescent horseradish peroxidase substrate (Millipore, WBKLS0100) was added to detect the antibody. The signal was captured with e-BLOT TOUCH IMAGER (Beijing Poliness Trade Co., Limited).

\section{Computational analyses of the disome-seq data for human cells}

The human genome (Ensembl Genes 101) was downloaded from Ensembl [87]. The most reliable principal isoform was used as the reference cDNA for each gene. The disome-seq data for human cells were retrieved from the Gene Expression Omnibus (GEO) at the National Center for Biotechnology Information (NCBI) under the accession number of GSE145723. The sequencing reads were mapped against human rRNAs with bowtie V1.2.2, and the unmapped reads were used in the following analyses. These reads were aligned against human protein-coding transcripts using STAR V2.7.3a [88] with the following parameters, --alignEndsType EndToEnd, --outFilterType BySJout, --outFilterMismatchNmax 2, --outFilterMultimapNmax 5, --outFilterMatchNmin 16. We followed the previous definition for the A-site of the leading ribosome in a disome footprint [38]. Specifically, offsets were $45 \mathrm{nts}$ for the 51-nt and 59-nt footprints, $46 \mathrm{nts}$ for the 52-nt and 60-nt footprints, $48 \mathrm{nts}$ for the 54-nt and 62-nt footprints, and 49 nts for the 55-nt and 63-nt footprints.

\section{The annotation of a-helices}

The protein secondary structure annotation of yeast protein was retrieved from UniProt [89] (https://www.uniprot.org/). Adjacent $\alpha$-helices without a gap were concatenated. Short $\alpha$-helices composed of 4 amino acids or less were filtered. The secondary structure of Rps31p was predicted with the online secondary structure prediction method, GOR IV [90], at https://npsa-prabi.ibcp.fr/.

\section{Alignment of proteins from fungi, animals, and plants}

Homologous genes were identified by BLAST (https://blast.ncbi.nlm.nih.gov/Blast.cgi). Orthologous gene pairs between yeast and human were retrieved from Ensembl BioMart [91] and NCBI HomoloGene [92]. Protein sequences were aligned by Clustal X [93]. Species trees were constructed with TimeTree [94] at http://www.timetree.org.

\section{Prediction of the minimum free energy of an mRNA fragment}

The minimum free energy of the 30-nt mRNA downstream of a disome footprint was calculated with the RNAfold V2.4.13 of the Vienna RNA package [95] (http://rna.tbi. univie.ac.at/).

\section{Supplementary Information}

The online version contains supplementary material available at https://doi.org/10.1186/s13059-020-02256-0. 
S4. The A-site pausing scores between replicates or between data processing approaches. Fig. S5. Highly translated mRNAs are crowded with ribosomes. Fig. S6. The contact interfaces of disomes (cycloheximide omitted in the lysate) and di-ribosomes. Fig. S7. The identification of disome-associated proteins. Fig. S8. Ribosome footprints in the two-ribosome-containing transcripts. Fig. S9. The detection of the $5^{\prime}$-ligation bias during the library preparation for monosome-seq and disome-seq. Fig. S10. The pausing scores estimated using the codon/aminoacid frequency in the genome as the background. Fig. S11. Additional cryo-EM structures of disomes. Fig. S12. On the 61-nt disome footprints. Fig. S13. On the 53-nt disome footprints. Fig. S14. The "inverse ramp" of disome footprints on the CDS. Fig. S15. The mRNA secondary structure downstream of disome footprints. Fig. S16. The presumed conformation and the putative A-site for disome footprints of various lengths Fig. S17. Cryo-EM data processing for disome particles that were collected with cycloheximide omitted in the lysis buffer. Fig. S18. Fourier shell correlation (FSC) curves for the final 3D density maps after RELION-based post-processing. Table S1. Summary of the monosome-seq libraries. Table S2. Summary of the disome-seq libraries. Table S3. Summary of the mRNA-seq libraries. Table S4. Disome-associated proteins (replicate 1: heavy isotope labeled disome proteins, disome/monosome intensity ratio > 1.5). Table S5. Disome-associated proteins (replicate 2: light isotope labeled disome proteins, disome/monosome intensity ratio > 1.5). Table S6. Primers used for tagging 4XFLAG to the Cterminus of each chaperone.

Additional file 2. Review history.

\section{Acknowledgements}

We thank Lucas Carey (Peking University) for commenting on and editing the manuscript. We thank Xiahe Huang and Yingchun Wang (IGDB, CAS) for technical support in proteomics, Ting Li (IGDB, CAS) in ribosome profiling, Rongxin Yang (IGDB, CAS) in molecular biological experiments, and Shaohuan Wu (IGDB, CAS), Yuliang Zhang (IGDB, CAS), and Haotian Guo (Huazhong University of Science and Technology) in computational biology. We thank Jinzhong Lin (Fudan University) and Xiaofeng Cao (IGDB, CAS) for discussion. We thank the Core Facilities at the School of Life Sciences, Peking University for help with negative staining EM, the Electron Microscopy Laboratory and the Cryo-EM Platform of Peking University for help with data collection, the High Performance Computing Platform of Peking University for help with computation, and the National Centre for Protein Sciences at Peking University for assistance with sample preparation. We also thank Addgene (http://www.addgene.org/) for providing plasmids.

\section{Review history}

The review history is available as Additional file 2.

\section{Peer review information}

Barbara Cheifet and Yixin Yao were the primary editors on this article and managed its editorial process and peer review in collaboration with the rest of the editorial team.

\section{Authors' contributions}

T.Z., N.G., and W.Q. designed the experiments. T.Z. performed disome-seq, mRNA-seq, and molecular biological experiments. T.Z. and J.W. performed monosome-seq. T.Z. and S.C. prepared the samples for MS/MS. Y.-M.C. performed data analyses for the above-mentioned experiments. Y.L., T.Z., and N.G. performed cryo-EM and the corresponding data analysis. T.Z., Y.-M.C., N.G., and W.Q. wrote the manuscript. The authors read and approved the final manuscript.

\section{Funding}

This work was supported by grants from the National Key R\&D Program of China (2019YFA0508700), the National Natural Science Foundation of China (31900455 to T.Z., 31725007 and 31630087 to N.G., and 31922014 to W.Q.), and the Youth Innovation Promotion Association of CAS (2020099).

\section{Availability of data and materials}

The S. cerevisiae genome (SGD R64-1-1) was downloaded from https://www.yeastgenome.org/ [75]. The protein secondary structure annotation of yeast protein was retrieved from UniProt (https://www.uniprot.org/) [89]. The human genome (Ensembl Genes 101) was retrieved from Ensembl [87]. Orthologous gene pairs between yeast and human were retrieved from Ensembl BioMarts [91] and NCBI HomoloGene [92].

The high-throughput sequencing data of disome-seq, monosome-seq, and mRNA-seq discussed in this publication have been deposited in NCBI GEO [96] and are accessible through GEO Series accession number GSE158572 [97]. The mass spectrometry proteomics data have been deposited to the ProteomeXchange Consortium (http:// proteomecentral.proteomexchange.org) via the PRIDE partner repository [98] with the dataset identifier PXD015271 [99]. The cryo-EM density maps have been deposited in the Electron Microscopy Data Bank (EMDB) (http://www. emdataresource.org/) [100] under accession numbers of EMD-30553 [101] and EMD-30554 [102] (the leading and trailing ribosomes of disome collected without cycloheximide in the lysis buffer) and EMD-30580 [103] and EMD-30581 [104] (the leading and trailing ribosomes of disome collected with cycloheximide in the lysis buffer). Codes to analyze the data are available at GitHub, under the terms of the MIT license [105], and at Zenodo [106].

\section{Ethics approval and consent to participate}

Not applicable.

\section{Consent for publication}

Not applicable.

\section{Competing interests}

The authors declare no competing interests. 


\section{Author details}

'State Key Laboratory of Plant Genomics, Institute of Genetics and Developmental Biology, Innovation Academy for Seed Design, Chinese Academy of Sciences, Beijing 100101, China. ${ }^{2}$ Key Laboratory of Genetic Network Biology, Institute of Genetics and Developmental Biology, Innovation Academy for Seed Design, Chinese Academy of Sciences, Beijing 100101, China. ${ }^{3}$ University of Chinese Academy of Sciences, Beijing 100049, China. ${ }^{4}$ Peking University-Tsinghua University-National Institute of Biological Sciences Joint Graduate Program, School of Life Science, Tsinghua University, Beijing 100084, China. ${ }^{5}$ State Key Laboratory of Membrane Biology, Peking-Tsinghua Center for Life Sciences, School of Life Sciences, Peking University, Beijing 100871, China.

\section{Received: 23 April 2020 Accepted: 20 December 2020}

\section{Published online: 05 January 2021}

\section{References}

1. Chu D, Kazana E, Bellanger N, Singh T, Tuite MF, von der Haar T. Translation elongation can control translation initiation on eukaryotic mRNAs. EMBO J. 2014;33:21-34.

2. Stein KC, Frydman J. The stop-and-go traffic regulating protein biogenesis: how translation kinetics controls proteostasis. J Biol Chem. 2019;294:2076-84.

3. Zhao TL, Zhang S, Qian WF. Cis-regulatory mechanisms and biological effects of translation elongation. Yi Chuan. 2020; 42:613-31.

4. Agashe D, Martinez-Gomez NC, Drummond DA, Marx CJ. Good codons, bad transcript: large reductions in gene expression and fitness arising from synonymous mutations in a key enzyme. Mol Biol Evol. 2013;30:549-60.

5. Sharma AK, O'Brien EP. Non-equilibrium coupling of protein structure and function to translation-elongation kinetics. Curr Opin Struct Biol. 2018:49:94-103.

6. Gershenson A, Gierasch LM. Protein folding in the cell: challenges and progress. Curr Opin Struct Biol. 2011;21:32-41.

7. Drummond DA, Wilke CO. Mistranslation-induced protein misfolding as a dominant constraint on coding-sequence evolution. Cell. 2008;134:341-52.

8. Richter JD, Coller J. Pausing on polyribosomes: make way for elongation in translational control. Cell. 2015;163:292-300.

9. Ikeuchi K, Tesina P, Matsuo Y, Sugiyama T, Cheng J, Saeki Y, Tanaka K, Becker T, Beckmann R, Inada T. Collided ribosomes form a unique structural interface to induce Hel2-driven quality control pathways. EMBO J. 2019;38:e100276.

10. Juszkiewicz S, Hegde RS. Initiation of quality control during poly(A) translation requires site-specific ribosome ubiquitination. Mol Cell. 2017;65:743-50 e744.

11. Sundaramoorthy E, Leonard M, Mak R, Liao J, Fulzele A, Bennett EJ. ZNF598 and RACK1 regulate mammalian ribosomeassociated quality control function by mediating regulatory 40S ribosomal ubiquitylation. Mol Cell. 2017;65:751-60 e754.

12. Park H, Subramaniam AR. Inverted translational control of eukaryotic gene expression by ribosome collisions. PLoS Biol. 2019;17:e3000396.

13. Brandman O, Hegde RS. Ribosome-associated protein quality control. Nat Struct Mol Biol. 2016;23:7-15.

14. Joazeiro CAP. Mechanisms and functions of ribosome-associated protein quality control. Nat Rev Mol Cell Biol. 2019;20: 368-83.

15. Matsuo Y, Tesina P, Nakajima S, Mizuno M, Endo A, Buschauer R, Cheng J, Shounai O, Ikeuchi K, Saeki Y, et al. RQT complex dissociates ribosomes collided on endogenous RQC substrate SDD1. Nat Struct Mol Biol. 2020;27:323-32.

16. Juszkiewicz S, Chandrasekaran V, Lin Z, Kraatz S, Ramakrishnan V, Hegde RS. ZNF598 is a quality control sensor of collided ribosomes. Mol Cell. 2018;72:469-81 e467.

17. Ingolia NT, Ghaemmaghami S, Newman JR, Weissman JS. Genome-wide analysis in vivo of translation with nucleotide resolution using ribosome profiling. Science. 2009;324:218-23.

18. Ingolia NT. Ribosome profiling: new views of translation, from single codons to genome scale. Nat Rev Genet. 2014;15: 205-13.

19. Hussmann JA, Patchett S, Johnson A, Sawyer S, Press WH. Understanding biases in ribosome profiling experiments reveals signatures of translation dynamics in yeast. PLoS Genet. 2015;11:e1005732.

20. Weinberg DE, Shah P, Eichhorn SW, Hussmann JA, Plotkin JB, Bartel DP. Improved ribosome-footprint and mRNA measurements provide insights into dynamics and regulation of yeast translation. Cell Rep. 2016;14:1787-99.

21. Qian W, Yang JR, Pearson NM, Maclean C, Zhang J. Balanced codon usage optimizes eukaryotic translational efficiency. Plos Genet. 2012:8:e1002603.

22. Charneski CA, Hurst LD. Positively charged residues are the major determinants of ribosomal velocity. Plos Biol. 2013;11: e1001508.

23. Yang JR, Chen X, Zhang J. Codon-by-codon modulation of translational speed and accuracy via mRNA folding. Plos Biol. 2014;12:e1001910.

24. Guydosh NR, Green R. Dom34 rescues ribosomes in 3' untranslated regions. Cell. 2014;156:950-62.

25. Ivanov IP, Shin BS, Loughran G, Tzani I, Young-Baird SK, Cao C, Atkins JF, Dever TE. Polyamine control of translation elongation regulates start site selection on antizyme inhibitor mRNA via ribosome queuing. Mol Cell. 2018;70:254-64 e256.

26. Diament A, Feldman A, Schochet E, Kupiec M, Arava Y, Tuller T. The extent of ribosome queuing in budding yeast. Plos Comput Biol. 2018;14:e1005951.

27. Wruck F, Katranidis A, Nierhaus KH, Buldt G, Hegner M. Translation and folding of single proteins in real time. Proc Natl Acad Sci U S A. 2017;114:E4399-407.

28. Hershberg R, Petrov DA. Selection on codon bias. Annu Rev Genet. 2008;42:287-99.

29. Nissley DA, O'Brien EP. Altered co-translational processing plays a role in Huntington's pathogenesis-a hypothesis. Front Mol Neurosci. 2016;9:54.

30. Clarke TF, Clark PL. Rare codons cluster. Plos One. 2008;3:e3412.

31. Chen S, Li K, Cao W, Wang J, Zhao T, Huan Q, Yang YF, Wu S, Qian W. Codon-resolution analysis reveals a direct and context-dependent impact of individual synonymous mutations on mRNA level. Mol Biol Evol. 2017;34:2944-58.

32. Yu CH, Dang Y, Zhou Z, Wu C, Zhao F, Sachs MS, Liu Y. Codon usage influences the local rate of translation elongation to regulate co-translational protein folding. Mol Cell. 2015;59:744-54. 
33. Arava Y, Wang Y, Storey JD, Liu CL, Brown PO, Herschlag D. Genome-wide analysis of mRNA translation profiles in Saccharomyces cerevisiae. Proc Natl Acad Sci U S A. 2003;100:3889-94.

34. Klopotowski T, Wiater A. Synergism of aminotriazole and phosphate on the inhibition of yeast imidazole glycerol phosphate dehydratase. Arch Biochem Biophys. 1965;112:562-6.

35. Patel BK, Gavin-Smyth J, Liebman SW. The yeast global transcriptional co-repressor protein Cyc8 can propagate as a prion. Nat Cell Biol. 2009;11:344-9.

36. Fang NN, Chan GT, Zhu M, Comyn SA, Persaud A, Deshaies RJ, Rotin D, Gsponer J, Mayor T. Rsp5/Nedd4 is the main ubiquitin ligase that targets cytosolic misfolded proteins following heat stress. Nat Cell Biol. 2014;16:1227-37.

37. Weinert BT, Scholz C, Wagner SA, lesmantavicius V, Su D, Daniel JA, Choudhary C. Lysine succinylation is a frequently occurring modification in prokaryotes and eukaryotes and extensively overlaps with acetylation. Cell Rep. 2013;4:842-51.

38. Han P, Shichino Y, Schneider-Poetsch T, Mito M, Hashimoto S, Udagawa T, Kohno K, Yoshida M, Mishima Y, Inada T, Iwasaki S. Genome-wide survey of ribosome collision. Cell Rep. 2020;31:107610.

39. Hedges SB, Dudley J, Kumar S. TimeTree: a public knowledge-base of divergence times among organisms. Bioinformatics. 2006;22:2971-2.

40. Rodnina MV. The ribosome in action: tuning of translational efficiency and protein folding. Protein Sci. 2016;25:1390-406

41. Nelson DL, Lehninger AL, Cox MM. Lehninger principles of biochemistry. 5th ed. New York: W. H. Freeman; 2008.

42. Bryson JW, Betz SF, Lu HS, Suich DJ, Zhou HX, O'Neil KT, DeGrado WF. Protein design: a hierarchic approach. Science. 1995;270:935-41.

43. Myers JK, Pace CN, Scholtz JM. Helix propensities are identical in proteins and peptides. Biochemistry. 1997;36:10923-9.

44. Chandrasekaran V, Juszkiewicz S, Choi J, Puglisi JD, Brown A, Shao S, Ramakrishnan V, Hegde RS. Mechanism of ribosome stalling during translation of a poly(A) tail. Nat Struct Mol Biol. 2019;26:1132-40.

45. Sharp PM, Li WH. The codon adaptation index--a measure of directional synonymous codon usage bias, and its potential applications. Nucleic Acids Res. 1987;15:1281-95.

46. Joazeiro CAP. Ribosomal stalling during translation: providing substrates for ribosome-associated protein quality control. Annu Rev Cell Dev Biol. 2017;33:343-68.

47. Matsuo Y, Ikeuchi K, Saeki Y, Iwasaki S, Schmidt C, Udagawa T, Sato F, Tsuchiya H, Becker T, Tanaka K, et al. Ubiquitination of stalled ribosome triggers ribosome-associated quality control. Nat Commun. 2017;8:159.

48. Ikeuchi K, Izawa T, Inada T. Recent progress on the molecular mechanism of quality controls induced by ribosome stalling. Front Genet. 2018;9:743.

49. Doring K, Ahmed N, Riemer T, Suresh HG, Vainshtein Y, Habich M, Riemer J, Mayer MP, O'Brien EP, Kramer G, Bukau B. Profiling Ssb-nascent chain interactions reveals principles of Hsp70-assisted folding. Cell. 2017;170:298-311 e220.

50. Fuchs RT, Sun Z, Zhuang F, Robb GB. Bias in ligation-based small RNA sequencing library construction is determined by adaptor and RNA structure. Plos One. 2015;10:e0126049.

51. Lareau LF, Hite DH, Hogan GJ, Brown PO. Distinct stages of the translation elongation cycle revealed by sequencing ribosome-protected mRNA fragments. Elife. 2014;3:e01257.

52. Mohammad F, Green R, Buskirk AR. A systematically-revised ribosome profiling method for bacteria reveals pauses at single-codon resolution. Elife. 2019:8:e42591.

53. Wu CC, Zinshteyn B, Wehner KA, Green R. High-resolution ribosome profiling defines discrete ribosome elongation states and translational regulation during cellular stress. Mol Cell. 2019;73:959-70 e955.

54. Mohammad F, Woolstenhulme CJ, Green R, Buskirk AR. Clarifying the translational pausing landscape in bacteria by ribosome profiling. Cell Rep. 2016;14:686-94.

55. McGlincy NJ, Ingolia NT. Transcriptome-wide measurement of translation by ribosome profiling. Methods. 2017;126:112-29.

56. Meydan S, Guydosh NR. Disome and trisome profiling reveal genome-wide targets of ribosome quality control. Mol Cell. 2020;79:588-602 e586.

57. Arpat AB, Liechti A, De Matos M, Dreos R, Janich P, Gatfield D. Transcriptome-wide sites of collided ribosomes reveal principles of translational pausing. Genome Res. 2020;30:985-99.

58. Prabhakar A, Capece MC, Petrov A, Choi J, Puglisi JD. Post-termination ribosome intermediate acts as the gateway to ribosome recycling. Cell Rep. 2017;20:161-72.

59. Presnyak V, Alhusaini N, Chen YH, Martin S, Morris N, Kline N, Olson S, Weinberg D, Baker KE, Graveley BR, Coller J. Codon optimality is a major determinant of mRNA stability. Cell. 2015;160:1111-24.

60. Aitken CE, Puglisi JD. Following the intersubunit conformation of the ribosome during translation in real time. Nat Struct Mol Biol. 2010;17:793-800.

61. Pelechano V, Wei W, Steinmetz LM. Widespread co-translational RNA decay reveals ribosome dynamics. Cell. 2015;161: 1400-12.

62. Tuller T, Carmi A, Vestsigian K, Navon S, Dorfan Y, Zaborske J, Pan T, Dahan O, Furman I, Pilpel Y. An evolutionarily conserved mechanism for controlling the efficiency of protein translation. Cell. 2010;141:344-54.

63. Schneider-Poetsch T, Ju J, Eyler DE, Dang Y, Bhat S, Merrick WC, Green R, Shen B, Liu JO. Inhibition of eukaryotic translation elongation by cycloheximide and lactimidomycin. Nat Chem Biol. 2010;6:209-17.

64. Archer SK, Shirokikh NE, Beilharz TH, Preiss T. Dynamics of ribosome scanning and recycling revealed by translation complex profiling. Nature. 2016;535:570-4.

65. Williams AJ, Paulson HL. Polyglutamine neurodegeneration: protein misfolding revisited. Trends Neurosci. 2008;31:521-8

66. Rauscher S, Baud S, Miao M, Keeley FW, Pomes R. Proline and glycine control protein self-organization into elastomeric or amyloid fibrils. Structure. 2006;14:1667-76.

67. Nilsson OB, Hedman R, Marino J, Wickles S, Bischoff L, Johansson M, Muller-Lucks A, Trovato F, Puglisi JD, O'Brien EP, et al. Cotranslational protein folding inside the ribosome exit tunnel. Cell Rep. 2015;12:1533-40.

68. Fedyukina DV, Cavagnero S. Protein folding at the exit tunnel. Annu Rev Biophys. 2011;40:337-59.

69. Voss NR, Gerstein M, Steitz TA, Moore PB. The geometry of the ribosomal polypeptide exit tunnel. J Mol Biol. 2006;360: 893-906.

70. Stein KC, Kriel A, Frydman J. Nascent polypeptide domain topology and elongation rate direct the cotranslational hierarchy of Hsp70 and TRiC/CCT. Mol Cell. 2019;75:1117-30 e1115. 
71. Chotewutmontri P, Barkan A. Dynamics of chloroplast translation during chloroplast differentiation in maize. Plos Genet. 2016;12:e1006106.

72. Bazzini AA, Lee MT, Giraldez AJ. Ribosome profiling shows that miR-430 reduces translation before causing mRNA decay in zebrafish. Science. 2012;336:233-7.

73. Chen J, Brunner AD, Cogan JZ, Nunez JK, Fields AP, Adamson B, Itzhak DN, Li JY, Mann M, Leonetti MD, Weissman JS. Pervasive functional translation of noncanonical human open reading frames. Science. 2020;367:1140-6.

74. Martin M. Cutadapt removes adapter sequences from high-throughput sequencing reads. EMBnet J. 2011;17:10-2.

75. Cherry JM, Hong EL, Amundsen C, Balakrishnan R, Binkley G, Chan ET, Christie KR, Costanzo MC, Dwight SS, Engel SR, et al. Saccharomyces Genome Database: the genomics resource of budding yeast. Nucleic Acids Res. 2012;40:D700-5.

76. Langmead B, Trapnell C, Pop M, Salzberg SL. Ultrafast and memory-efficient alignment of short DNA sequences to the human genome. Genome Biol. 2009;10:R25.

77. Kim D, Pertea G, Trapnell C, Pimentel H, Kelley R, Salzberg SL. TopHat2: accurate alignment of transcriptomes in the presence of insertions, deletions and gene fusions. Genome Biol. 2013;14:R36.

78. Lu J, Deutsch C. Folding zones inside the ribosomal exit tunnel. Nat Struct Mol Biol. 2005;12:1123-9.

79. Merritt GH, Naemi WR, Mugnier P, Webb HM, Tuite MF, von der Haar T. Decoding accuracy in eRF1 mutants and its correlation with pleiotropic quantitative traits in yeast. Nucleic Acids Res. 2010;38:5479-92.

80. Mastronarde DN. Automated electron microscope tomography using robust prediction of specimen movements. J Struct Biol. 2005;152:36-51.

81. Zheng SQ, Palovcak E, Armache JP, Verba KA, Cheng Y, Agard DA. MotionCor2: anisotropic correction of beam-induced motion for improved cryo-electron microscopy. Nat Methods. 2017;14:331-2.

82. Shaikh TR, Gao H, Baxter WT, Asturias FJ, Boisset N, Leith A, Frank J. SPIDER image processing for single-particle reconstruction of biological macromolecules from electron micrographs. Nat Protoc. 2008;3:1941-74.

83. Zhang K. Gctf: real-time CTF determination and correction. J Struct Biol. 2016;193:1-12.

84. Zivanov J, Nakane T, Forsberg BO, Kimanius D, Hagen WJ, Lindahl E, Scheres SH. New tools for automated highresolution cryo-EM structure determination in RELION-3. eLife. 2018;7:e42166.

85. Chen Y, Chen S, Li K, Zhang Y, Huang X, Li T, Wu S, Wang Y, Carey LB, Qian W. Overdosage of balanced protein complexes reduces proliferation rate in aneuploid cells. Cell Syst. 2019;9:129-42 e125.

86. Cox J, Mann M. MaxQuant enables high peptide identification rates, individualized p.p.b.-range mass accuracies and proteome-wide protein quantification. Nat Biotechnol. 2008;26:1367-72.

87. Yates AD, Achuthan P, Akanni W, Allen J, Allen J, Alvarez-Jarreta J, Amode MR, Armean IM, Azov AG, Bennett R, et al. Ensembl 2020. Nucleic Acids Res. 2020;48:D682-8.

88. Dobin A, Davis CA, Schlesinger F, Drenkow J, Zaleski C, Jha S, Batut P, Chaisson M, Gingeras TR. STAR: ultrafast universal RNA-seq aligner. Bioinformatics. 2013;29:15-21.

89. UniProt Consortium. UniProt: a worldwide hub of protein knowledge. Nucleic Acids Res. 2019;47:D506-15.

90. Garnier J, Gibrat JF, Robson B. GOR method for predicting protein secondary structure from amino acid sequence. Methods Enzymol. 1996;266:540-53.

91. Kinsella RJ, Kahari A, Haider S, Zamora J, Proctor G, Spudich G, Almeida-King J, Staines D, Derwent P, Kerhornou A, et al. Ensembl BioMarts: a hub for data retrieval across taxonomic space. Database (Oxford). 2011;2011:bar030.

92. Resource Coordinators NCBI. Database resources of the National Center for Biotechnology Information. Nucleic Acids Res. 2018;46:D8-D13.

93. Larkin MA, Blackshields G, Brown NP, Chenna R, McGettigan PA, McWilliam H, Valentin F, Wallace IM, Wilm A, Lopez R, et al. Clustal W and Clustal X version 2.0. Bioinformatics. 2007;23:2947-8.

94. Kumar S, Stecher G, Suleski M, Hedges SB. TimeTree: a resource for timelines, timetrees, and divergence times. Mol Biol Evol. 2017;34:1812-9.

95. Lorenz R, Bernhart SH, Honer Zu Siederdissen C, Tafer H, Flamm C, Stadler PF, Hofacker IL. ViennaRNA Package 2.0. Algorithms Mol Biol. 2011;6:26.

96. Barrett T, Wilhite SE, Ledoux P, Evangelista C, Kim IF, Tomashevsky M, Marshall KA, Phillippy KH, Sherman PM, Holko M, et al. NCBI GEO: archive for functional genomics data sets--update. Nucleic Acids Res. 2013;41:D991-5.

97. Zhao T, Chen Y-M, Li Y, Wang J, Chen S, Gao N, Qian W. Disome-seq data in yeast cells. Gene Expression Omnibus. 2020. https://www.ncbi.nlm.nih.gov/geo/query/acc.cgi?acc=GSE158572. Accessed 10 Dec 2020.

98. Perez-Riverol Y, Csordas A, Bai J, Bernal-Llinares M, Hewapathirana S, Kundu DJ, Inuganti A, Griss J, Mayer G, Eisenacher $\mathrm{M}$, et al. The PRIDE database and related tools and resources in 2019: improving support for quantification data. Nucleic Acids Res. 2019;47:D442-50.

99. Zhao T, Chen Y-M, Li Y, Wang J, Chen S, Gao N, Qian W. Disome-seq reveals sequence-mediated coupling of translational pauses and protein structures. ProteomeXchange Consortium. 2020. https://www.ebi.ac.uk/pride/archive/ projects/PXD015271. Accessed 10 Dec 2020.

100. Lawson CL, Baker ML, Best C, Bi C, Dougherty M, Feng P, van Ginkel G, Devkota B, Lagerstedt I, Ludtke SJ, et al. EMDataBank.org: unified data resource for CryoEM. Nucleic Acids Res. 2011;39:D456-64.

101. Zhao T, Chen Y-M, Li Y, Wang J, Chen S, Gao N, Qian W. Cryo-EM structure of disome from saccharomyces cerevisiae, the leading ribosome in the rotated state with A/P and P/E tRNAs. Electron Microscopy Data Bank. 2020. https://www. ebi.ac.uk/pdbe/entry/emdb/EMD-30553. Accessed 23 Dec 2020.

102. Zhao T, Chen Y-M, Li Y, Wang J, Chen S, Gao N, Qian W. Cryo-EM structure of disome from saccharomyces cerevisiae, the trailing ribosome in the rotated state with A/P and P/E tRNAs. Electron Microscopy Data Bank. 2020. https://www. ebi.ac.uk/pdbe/entry/emdb/EMD-30554. Accessed 23 Dec 2020.

103. Zhao T, Chen Y-M, Li Y, Wang J, Chen S, Gao N, Qian W. Cryo-EM structure of disome from saccharomyces cerevisiae, the leading ribosome in the rotated state with A/P and P/E tRNAs (cycloheximide treatment). Electron Microscopy Data Bank. 2020. https://www.ebi.ac.uk/pdbe/entry/emdb/EMD-30580. Accessed 23 Dec 2020.

104. Zhao T, Chen Y-M, Li Y, Wang J, Chen S, Gao N, Qian W. Cryo-EM structure of disome from saccharomyces cerevisiae, the trailing ribosome in the rotated state with A/P and P/E tRNAs (cycloheximide treatment). Electron Microscopy Data Bank. 2020. https://www.ebi.ac.uk/pdbe/entry/emdb/EMD-30581. Accessed 23 Dec 2020. 
105. Zhao T, Chen Y-M, Li Y, Wang J, Chen S, Gao N, Qian W. Disome-seq. Github. 2020. https://github.com/mingming-cgz/ Disome-seq. Accessed 10 Dec 2020.

106. Zhao T, Chen Y-M, Li Y, Wang J, Chen S, Gao N, Qian W. Disome-seq. Zenodo. 2020. https://doi.org/10.5281/zenodo. 4312535. Accessed 10 Dec 2020.

\section{Publisher's Note}

Springer Nature remains neutral with regard to jurisdictional claims in published maps and institutional affiliations.

Ready to submit your research? Choose BMC and benefit from:

- fast, convenient online submission

- thorough peer review by experienced researchers in your field

- rapid publication on acceptance

- support for research data, including large and complex data types

- gold Open Access which fosters wider collaboration and increased citations

- maximum visibility for your research: over $100 \mathrm{M}$ website views per year

At $\mathrm{BMC}$, research is always in progress.

Learn more biomedcentral.com/submissions 